Supporting information

\title{
Programming Dynamic Assembly of Viral Proteins with DNA Origami
}

\author{
Kun Zhou, ${ }^{\dagger \dagger}$ Yihao Zhou, ${ }^{\dagger}$ Victor Pan,,${ }^{\ddagger}$ Qiangbin Wang, ${ }^{*} \dagger, \S$ Yonggang $\mathrm{Ke}^{*}, \pitchfork$
}

†Key Laboratory of Nano-Bio Interface, Division of Nanobiomedicine and i-Lab, CAS Center for Excellence in Brain Science, Suzhou Institute of Nano-Tech and Nano-Bionics, Chinese Academy of Sciences, Suzhou 215123, China

*Wallace H. Coulter Department of Biomedical Engineering, Georgia Institute of Technology and Emory University, Atlanta, Georgia 30322, United States

${ }^{\S}$ School of Nano-Tech and Nano-Bionics, University of Science and Technology of China, Hefei 230026, China

*To whom correspondence should be addressed: qbwang2008@sinano.ac.cn; yonggang.ke@emory.edu 


\section{Materials and Methods}

\section{Preparation of DNA Origami Structures.}

DNA oligonucleotides were purchased from Sangon Biotech and Integrated DNA Technologies. M13mp18 single stranded DNA was purchased from New England Biolabs and the single-stranded DNA p8064 was purchased from Integrated DNA Technologies. Triangular DNA origami was assembled according to Rothemund's protocol ${ }^{[1]}$ in $1 \times$ TAE-Mg buffer (40 mM Tris, $20 \mathrm{mM}$ acetic acid, $2 \mathrm{mM}$ EDTA, $12.5 \mathrm{mM}$ magnesium acetate; $\mathrm{pH} 7.5$ ) and annealed by slowly cooling from $80^{\circ} \mathrm{C}$ to room temperature over $12 \mathrm{~h}$. 23-helix bundle (23HB) DNA barrel origami was fabricated by mixing the p8064 with staple strands in $\mathrm{pH} 8.0$ TE buffer containing $10 \mathrm{mM}$ Tris, $1 \mathrm{mM}$ EDTA, and $12 \mathrm{mM} \mathrm{MgCl}_{2}$. The folding reaction was carried out on a Thermocycler (Mastercycler Pro, Eppendorf) with isothermal annealing at $53^{\circ} \mathrm{C}$ for $48 \mathrm{~h}$. The DNA origami was purified by agarose gel electrophoresis in $0.5 \times$ TBE-Mg buffer and extracted via a Freeze 'N Squeeze spin column (Bio-Rad, USA). Then the buffer was exchanged by using a DEPC-treated Amicon centrifugal filter (100 kDa MWCO, $0.5 \mathrm{~mL}$, Merck Millipore), supplemented with buffer A (10 mM Tris, $1 \mathrm{mM}$ EDTA, $400 \mathrm{mM} \mathrm{NaCl}, 5 \mathrm{mM} \mathrm{MgCl}_{2}$; pH 6.9). The structures after purification were subjected to TEM imaging to verify quality. RNase inhibitor (RiboLock RNase Inhibitor, Thermo Scientific) was added into DNA origami solution to a final concentration of $0.5 \sim 1 \mathrm{U} / \mu \mathrm{L}$ unless otherwise stated.

\section{Preparation of RNA Strand and Viral Capsid Protein.}

The RNA Strand was carried out as previously described ${ }^{[2]}$. In brief, DNA template was obtained by PCR using a pair of primers (FP: 5'- GTAATACGACTCACTATAGGG -3', RP: 5'- TATGGTCGACCTGCAGGCGG -3') and pGEM-T/ML-RNA containing the target sequence. Then 1234-nt RNA transcripts were prepared using TranscriptAid T7 High Yield Transcription Kit (Thermo Scientific) according to the manufacturers' protocol. and purified by the standard procedure of phenol ( $\mathrm{pH} 4.7)$ : chloroform extraction and ethanol precipitation.

Capsid proteins were extracted from the native TMV virions using the acetic-acid-method as described by Fraenkel-Conrat ${ }^{[3]}$. The capsid proteins were finally dissolved in $75 \mathrm{mM}$ sodium potassium phosphate buffer $(\mathrm{pH}$ 7.2) and the concentration was measured using a NanoDrop spectrophotometer (Thermo Scientific) (determined by $\mathrm{A} 280=1.3$, per $1 \mu \mathrm{g} / \mu \mathrm{L}$ ). In order to induce $20 \mathrm{~S}$ disk formation, capsid proteins were incubated for at least $48 \mathrm{~h}$ at room temperature, while, incubation at a low temperature of 
$4^{\circ} \mathrm{C}$ was used to obtain small protein oligomers (the so-called 'A-protein' $)^{[4]}$.

\section{Dynamic Assembly of Protein Nanorod on DNA Origami.}

Typically, for routing RNA template on DNA origami scaffold, a $50 \mu \mathrm{L}$ of $4 \mathrm{nM}$ DNA origami was mixed with $0.5 \mu \mathrm{L}$ of $2 \mu \mathrm{M}$ RNA and annealed on thermocycler from $45^{\circ} \mathrm{C}$ to $41^{\circ} \mathrm{C}$ at $2 \mathrm{~min} /{ }^{\circ} \mathrm{C}$ followed by cooling from $40^{\circ} \mathrm{C}$ to $36^{\circ} \mathrm{C}$ at $5 \mathrm{~min} /{ }^{\circ} \mathrm{C}$, and then from $35^{\circ} \mathrm{C}$ to $26^{\circ} \mathrm{C}$ at $10 \mathrm{~min} /{ }^{\circ} \mathrm{C}$, for three cycles, followed by incubation at $16^{\circ} \mathrm{C}$ overnight. $1.5 \mu \mathrm{L}$ of capsid protein $(10 \mathrm{mg} / \mathrm{mL})$ in disk formation was added into the mixture to quickly activate assembly process and $1 \mu \mathrm{L}$ of capsid protein in small oligomer formation was added for the sake of subsequent routing assembly process. Aliquot was subjected to TEM characterization after incubation at room temperature for $1 \mathrm{~h}$. To control the stepwise assembly and motion upon the scheduled route, $0.4 \mu \mathrm{L}$ of premix solution containing each of $10 \mu \mathrm{M}$ of specific release strand was sequentially added to the mixture with each additional incubation of $2 \mathrm{~h}$. Aliquots of sample at different dynamic assembly stages were prepared for TEM characterization.

\section{Fluorescence Measurements.}

The fluorescence assays were performed on a fluorescence microplate reader Synergy H1 (BioTek) with an excitation wavelength of $492 \mathrm{~nm}$ and a detection emission wavelength of $522 \mathrm{~nm}$. For the detection of routing assembly at each phase, firstly, $40 \mu \mathrm{L}$ of the mixture containing the RNA-patterned triangular DNA origami $(2.5 \mathrm{nM}$, supplemented with RNase inhibitor to $1.8 \mathrm{U} / \mu \mathrm{L}$ ) and capsid protein was placed into a black 384 well plate (Corning) and a MicroAmp optical adhesive film (Applied Biosystems) was used to seal the microplate. Then, the fluorescence intensity of the sample corresponding to each stage by sequentially adding high-concentrated release strands was measured after an additional incubation time of $2 \mathrm{~h}$, and a control of denatured DNA origami with the same concentration was set for correction of possible photo-bleaching. For kinetics analysis, the time dependence of the intensity was monitored once every 30 seconds at $22^{\circ} \mathrm{C}$.

\section{Characterization of Routing RNA on Triangular DNA Origami Using AFM.}

For AFM characterization, the truncated capturing strands without the release-toehold region were employed to help pattern RNA strand on the triangular origami template. A $2 \mu \mathrm{L}$ of sample was dropped onto freshly cleaved mica surface. Then, a drop of $80 \mu \mathrm{L}$ of $1 \times$ TAE-Mg buffer was added into the liquid cell. Imaging was performed under tapping mode 
using a J scanner of a Multi-mode Nanoscope IIIa AFM (Vecco/Digital Instruments) with SNL tips. All recorded AFM images were processed and analyzed by NanoScope Analysis software.

\section{TEM Characterization.}

Sample solution was adsorbed to a carbon-coated copper grid, which was negatively glow discharged with a plasma cleaner, removed after 1 2 min with filter papers, and followed by rinsing by pure water and then negatively stained for 20 seconds with uranyl acetate or uranyl formate. Samples were imaged by using Tecnai G2 F20 S-Twin TEM operated at $200 \mathrm{kV}$ and Hitachi HT-7700 at a voltage of $80 \mathrm{kV}$. For each specimen, about 40 protein rods in designed hybrid structures were examined for length analysis using the ImageJ software. 


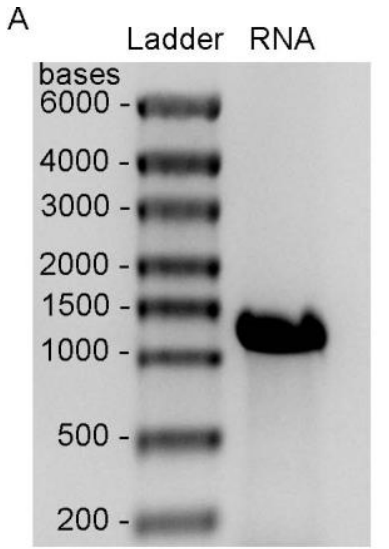

B

GGGCGAATTGGGCCCGAGCTTATTGATAGTGGATACGTCTGITTAGCCGGTTTGGTCGTCACGGGCGAGTG GAACTTGCCTGACAATTGCAGAGGAGGTGTGAGCGTGTGTCTGGTGGACAAAAGGATGGAAAGAGTCGAC GAGGCCACTCTCGGATCTTACTACACAGCAGCTGCAAAGAAAAGATTTCAGTTCAAGGTCGTTCCCAATTAT GCTATAACCACCCAGGACGCGATGAAAAACGTCTGGCAAGTITTAGTTAATGTTAGAAATGTGAAGATGTCA GCGGGTTTCTGTCCGCTTTCTCTGGAGTTTGTGTCGGTGTGTATTGTTTATAGAAATAATATAAAATTAGGTIT GAGAGAGAAGATTACAAACGTGAGAGACGGAGGGCCCATGGAACTTACAGAAGAAGTTGTTGATGAGTTC ATGGAAGATGTCCCTATGTCAATCAGGCTTGCAAAGTTTCGATCTCGAACCGGGAAAAGAGTGATGTCCGT AAGGGGAAAATTAGTAGTGGTGATCGGTTAGTGCCGAACAAGAACTATAGAAATGTTAAGGATTTTGGAGG AATGAGTTTTAAAAAGAATAATTTAATCGATGATGATTCGGAGGCTACTGTCGCCGAATCGGATTCGTTITAA ATATGTCTTATAGTATCACTACTCCATCTCAGTTCGTGTTCTTGTCATCAGCGTGGGCCGATCCAATAGAGTTAA TTAATATATGTACTAATGCCTTAGGAAATCAGTTTCAAACACAACAAGCTCGAACTGTCGTTCAAAGACAATT CAGTGAGGTGTGGAAACCTTCACCACAAGTAACTGTTAGGTTCCCTGACAGTGACTTTAAGGTGTACAGGT ACAATGCGGTATTAGACCCGCTAGTCACAGCACTGTTAGGTGCATTTGACACTAGAAATAGAATAATAGAAGT TGAAAATCAGGCGAACCCCACGACTGCCGAAACGTTAGATGCTACTCGTAGAGTAGACGACGCAACGGTGG CCACAAGGAGCGCGATAAATAATTTAATAGTAGAATTGATCAGAGGAACCGGATCTTACAATCGGAGCTCTT TCGAGAGCTCTTCTGGTTTGGTTTGGACCTCTAGTCCTGCAACTTGAGCTAGTCAAGATGCATAATAAATAAC GGATTGTGTCCGTAATCACACGTGGTGCGCTCGAATTCGTCTCTCCCAATCACTAGTGCGGCCGCCTGCAGG TCGACCATA

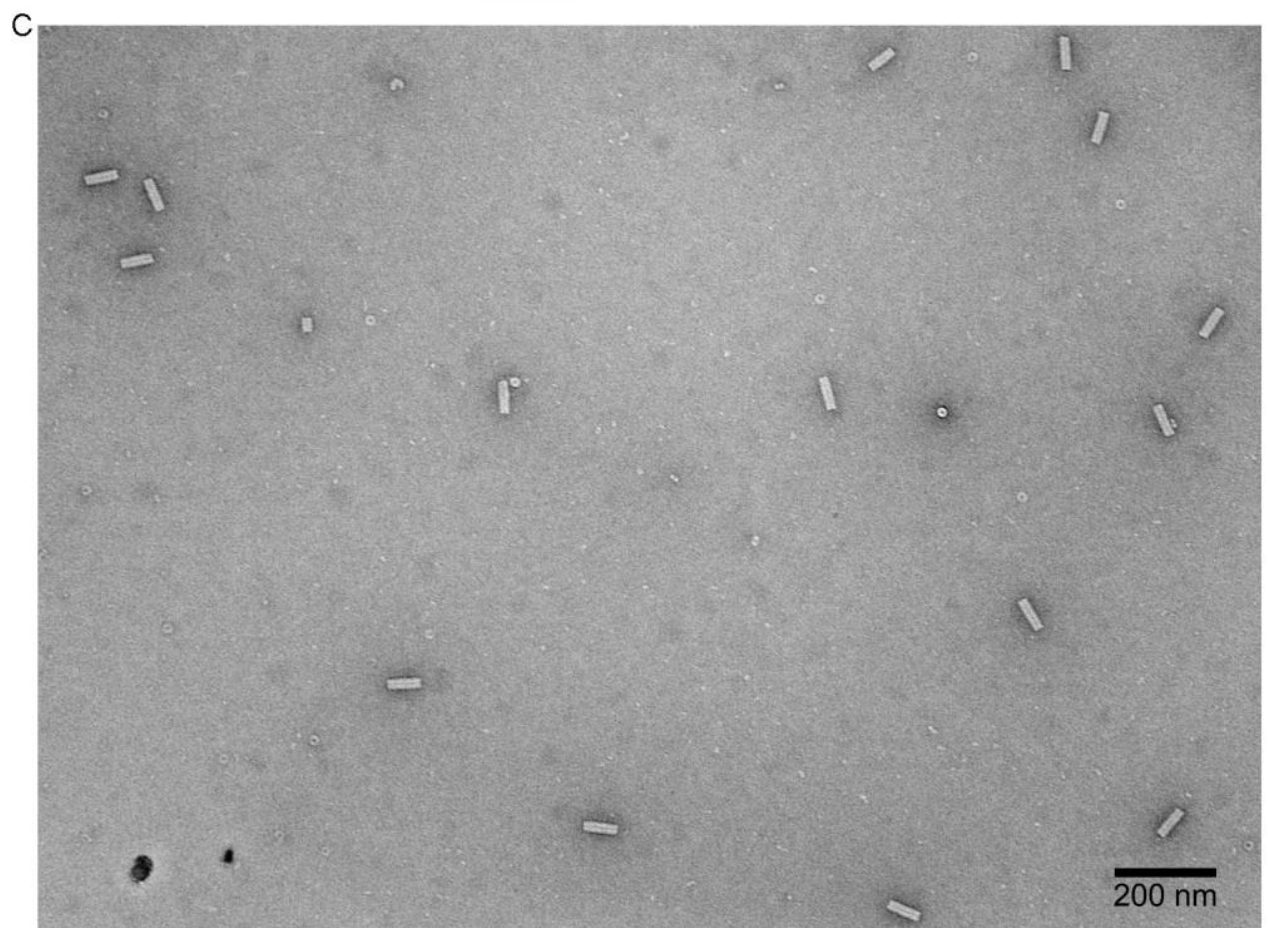

Figure S1. (A) Denaturing formaldehyde agarose gel electrophoresis for RNA quality assessment. Lane 1: RNA ladder with different size ranges. Lane 2: purified 1234-nt RNA transcripts. (B) Sequence information of the RNA strand, in which the core region of the TMV origin of the assembly sequence (OAS) is marked in red. (C) TEM image of the encapsidation of the 1234-nt RNA strand showing uniform protein nanorods. 


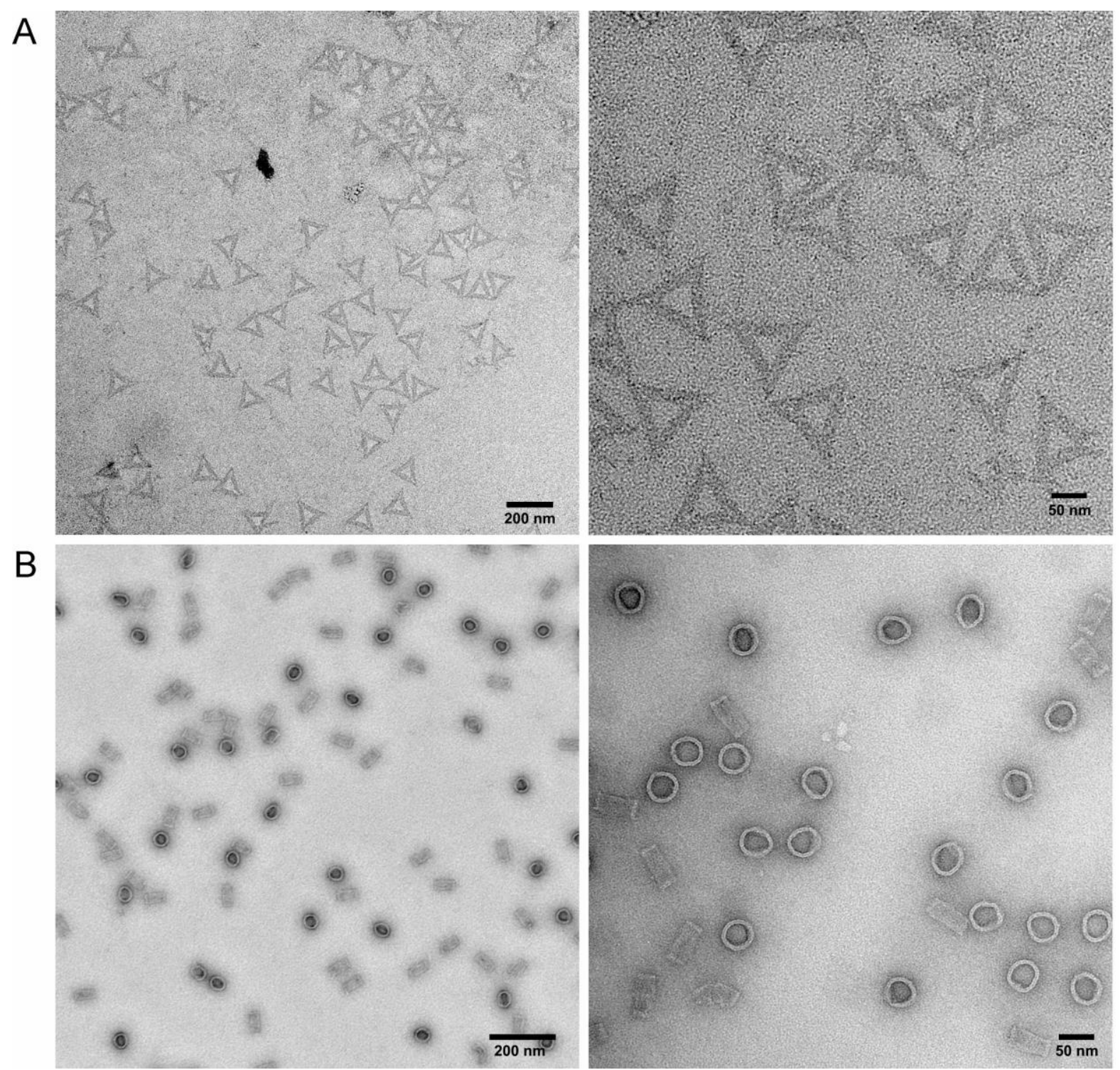

Figure S2. TEM images of the triangular DNA origami (A) and 23HB barrel DNA origami (B). 


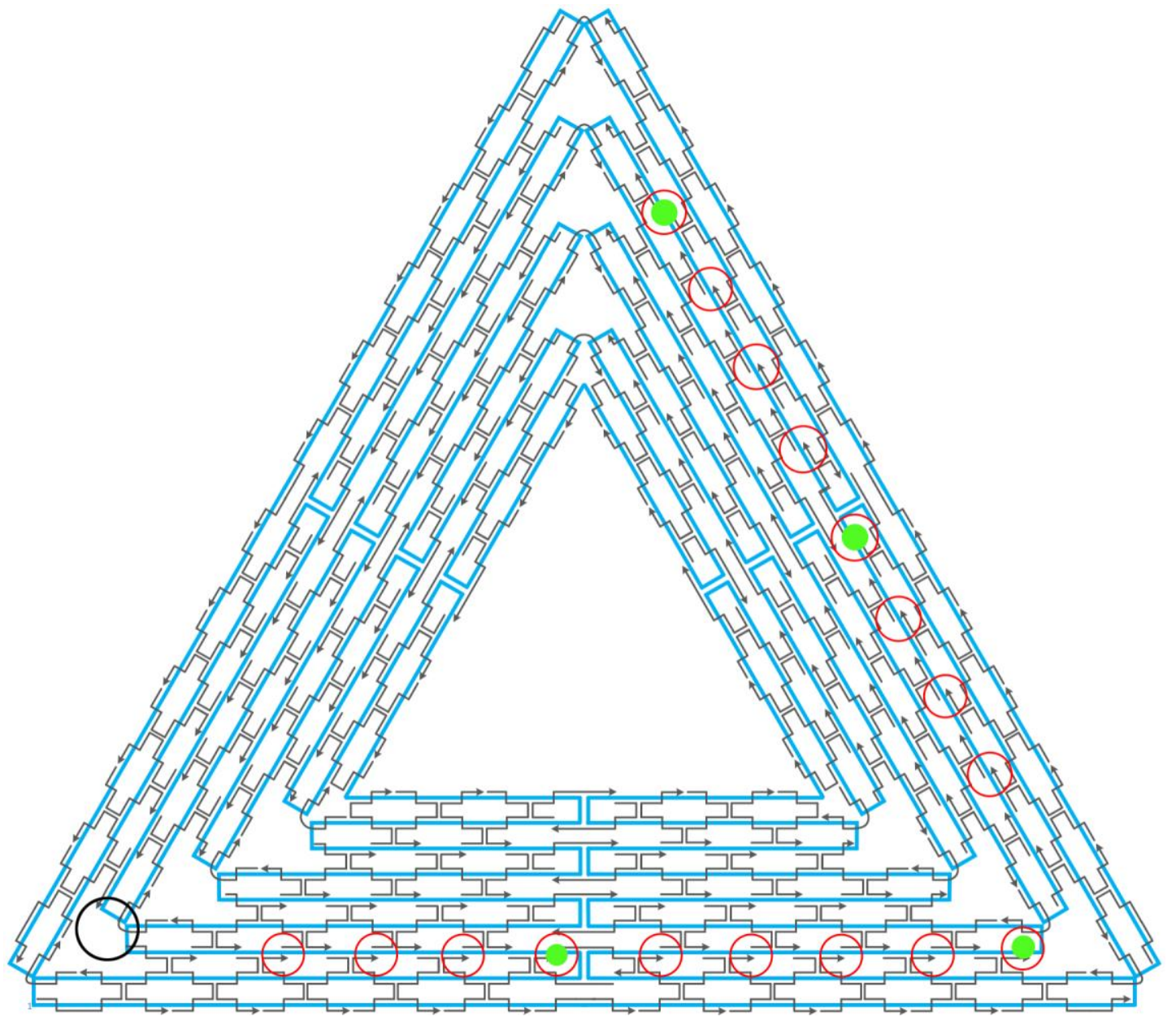

Figure S3. Strand diagram of triangle DNA origami design and the pattern design of RNA route. A total of 17 docking sites as path points are denoted with red circles, four of which marked with green dots inside are set as checkpoints with fluorophore-quencher pairs. The black circle indicates the anchor point for capturing the 3'-terminus of RNA. 


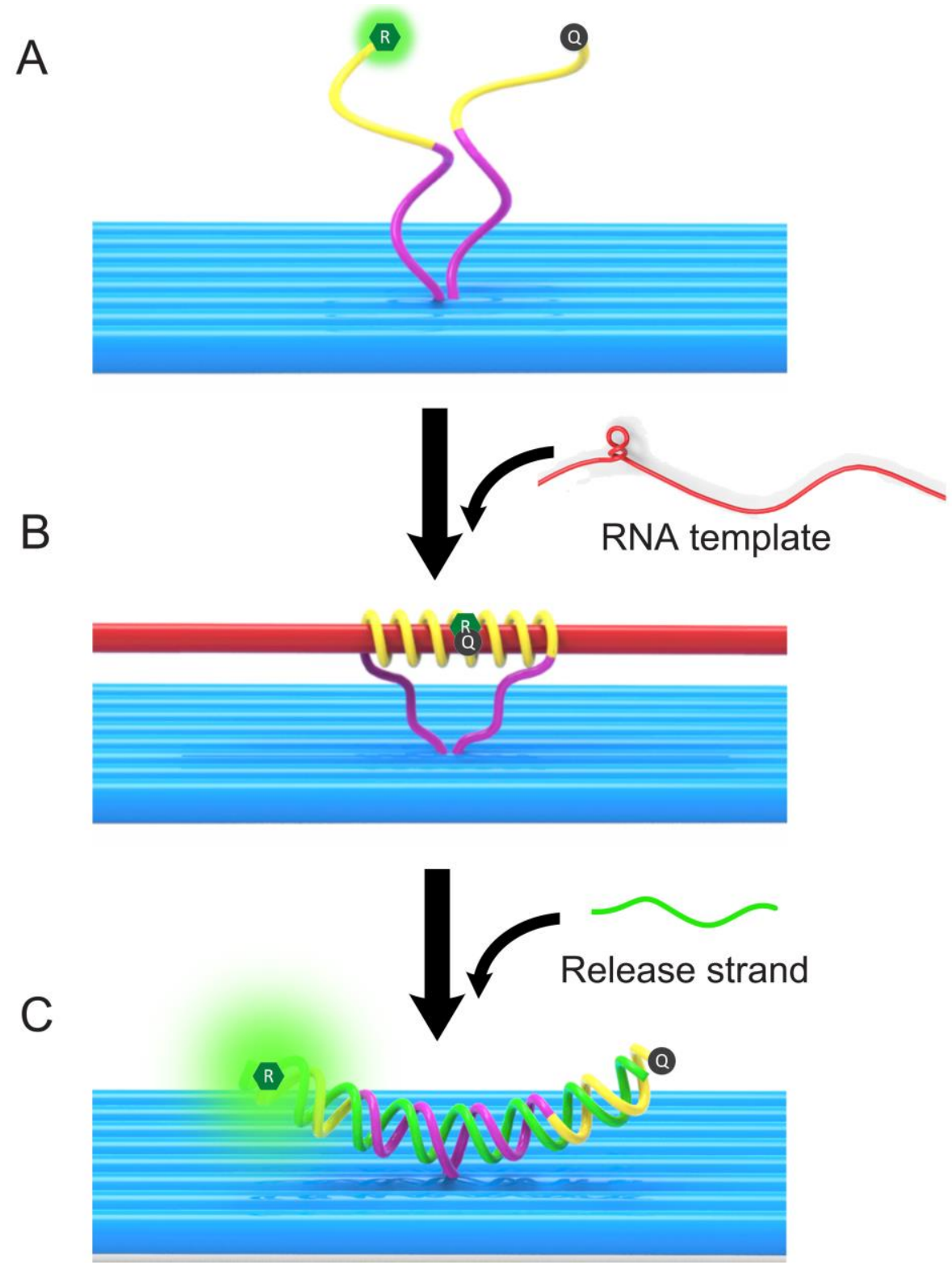

Figure S4. Schematic design of DNA strand exchange reaction to capture and release RNA on a path point as a fluorescence checkpoint. (A) A pair of routing strands each consisting the binding region (yellow) and release-toehold region (purple) is extended from the same position on a DNA origami scaffold (blue). Their terminals are labeled with a reporter dye of 5-FAM (dark green) and a quencher of BHQ-1 (black), respectively. (B) Two binding regions of the pair of routing strands are complementary to the successive domains of RNA and lock it, resulting in conformational change of the fluorophore-quencher pair into a closed state. (C) After addition of the specific release strands (green) that is full complementary to the pair of routing strands, the programmed toehold-mediated strand displacement reaction will release RNA from the path point and transform the fluorophore-quencher pair into a stiff open state $^{[5]}$, leading to an obvious increase of fluorescence signal. 

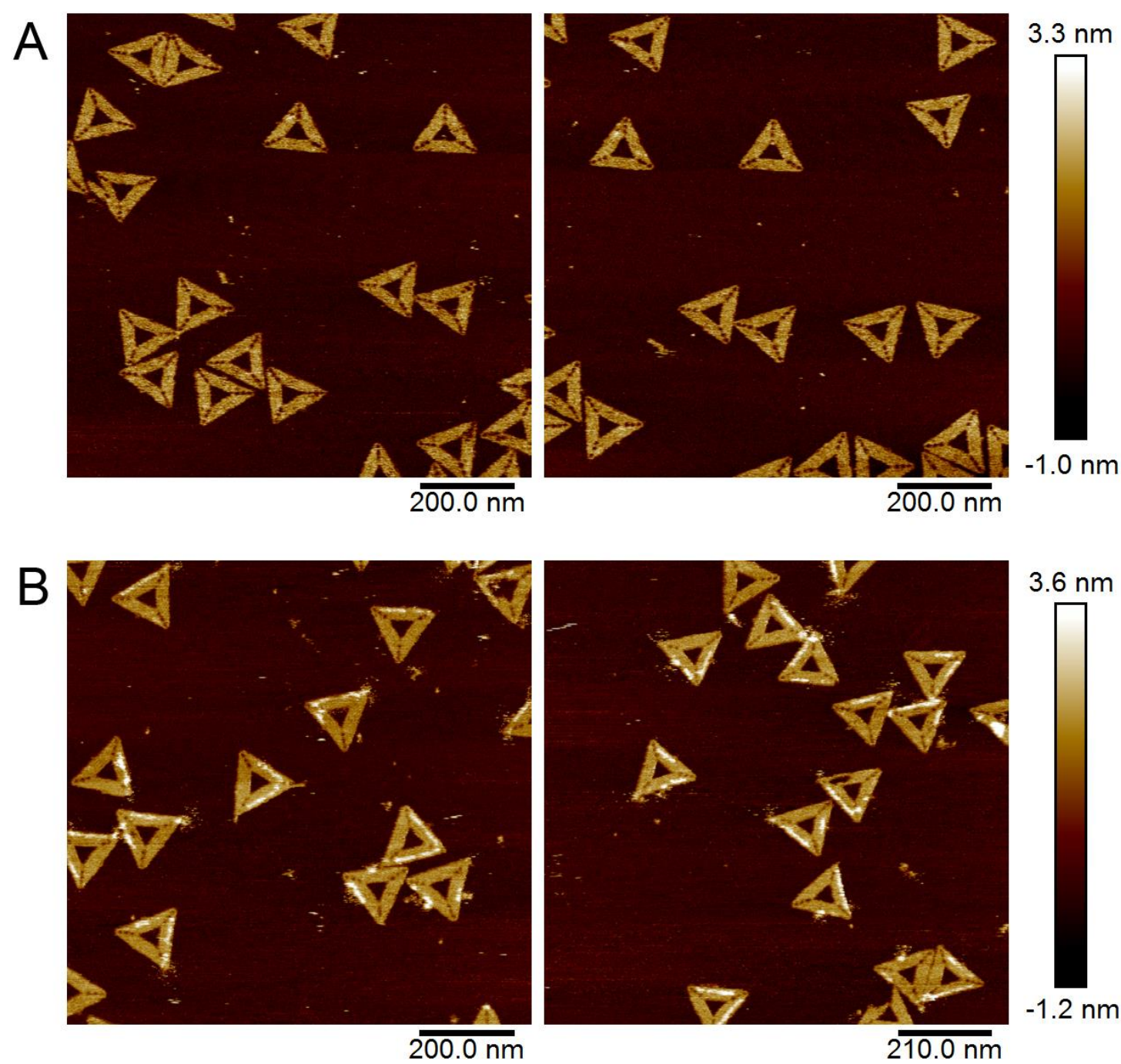

Figure S5. AFM images of triangular origami before (A) and after (B) hybridization with RNA. For sensitive detection of RNA on triangular origami using AFM, the 16 nt flexible segment as release-toehold region in each routing strand is removed, as the stiffening structure is readily sensed mechanically with an AFM cantilever. RNase inhibitor was not used in this experiment for the sake of reduction of background noise in AFM images. We assume the degradation of RNA due to contamination or scratch by AFM tip. 
To evaluate the binding capability of path points for capturing RNA, we designed and assembled four batch of triangle origami with different routing paths: one-quarter, half, three-quarter, and all 17 programmed path points. We used gel electrophoresis and AFM to detailly check the RNA binding efficiency. As shown in Figure S6, agarose gel results indicate that all four origami-RNA samples exhibit the slower band mobilities due to the banding of RNA, compared to the control sample of the triangle origami. We then did the liquid-phase AFM imaging in presence of RNase inhibitors. As shown in Figure S7-10, the AFM images indicate the binding zone of DNA strands on the one-quarter, half, three-quarter, and all path points can efficiently capture RNA, resulting in 77\%, 90\%, 75\%, $79 \%$ yield.

To further investigate the RNA binding efficiency, particularly whether potential RNA damage can happen in the assembly and reduce the binding efficiency, we examined the RNA binding to four anchor points by using the dye-quencher pairs, and compare the results with the DNA binding to these four anchor points. Four short DNA oligos with the sequences as same as the RNA binding sequences were used, and each DNA strand bind to one dye-quencher anchor point (Figure S11). We measured the fluorescence intensity of them (Figure S12). The gradually increased fluorescence values agree well with the numbers of dye-quencher pairs in open state. In phase v, all the path points should be blocked by the release strand and thus no DNA or RNA can bind onto the triangle origami, resulting in the highest fluorescence intensity. We used the quenching efficiencies to evaluate the binding. Comparing to the quenching efficiencies of DNA-DNA binding, we estimated the relatively binding efficiency of RNA-DNA are $78 \%, 79 \%, 94 \%, 110 \%$ for phase i, ii, iii, and iv, respectively. 


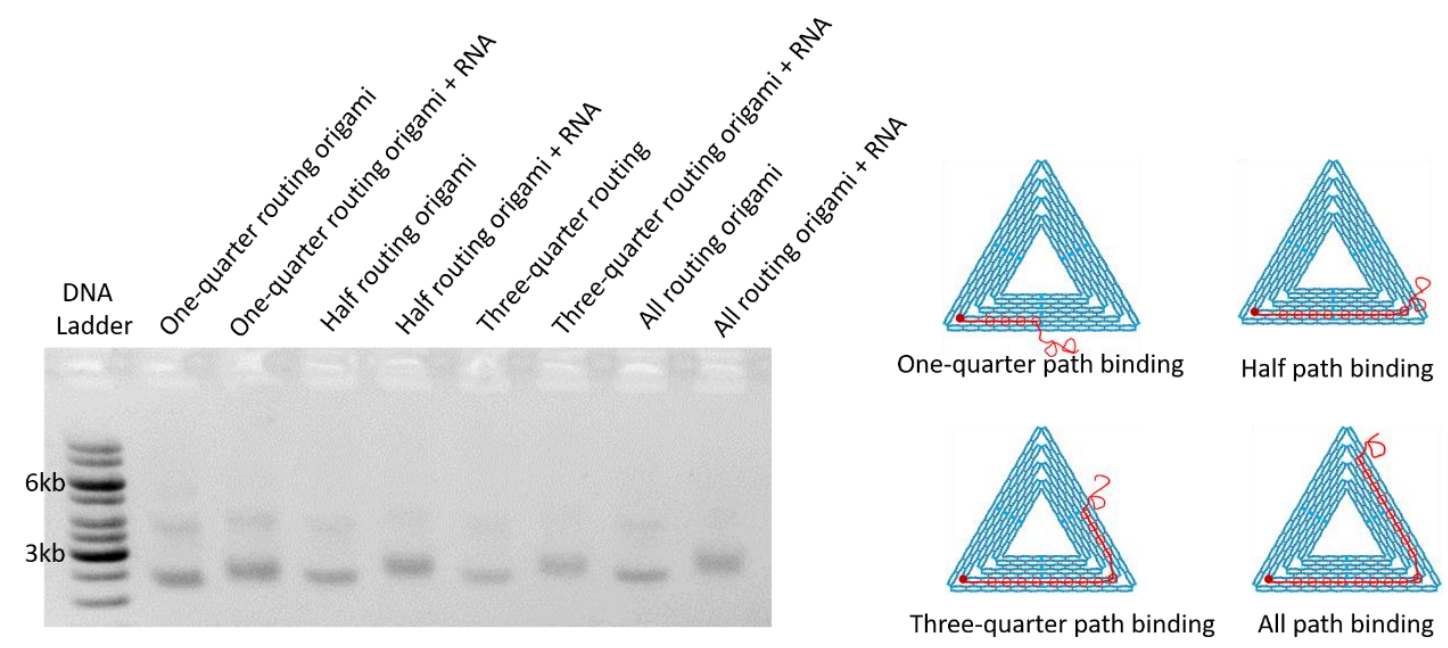

Figure S6. Agarose gel electrophoresis to evaluate the binding capability of path points with RNA. Left: Agarose gel result of DNA origami with one-quarter, half, three-quarter, and all path points and the corresponding DNA origami-RNA samples. Note that some upper dim bands occurred because of the formation of triangle origami dimer based on nonsticky end stacking. Right: four schematic drawings show the RNA binding on triangle origami with one-quarter, half, three-quarter, and all path points, respectively. 

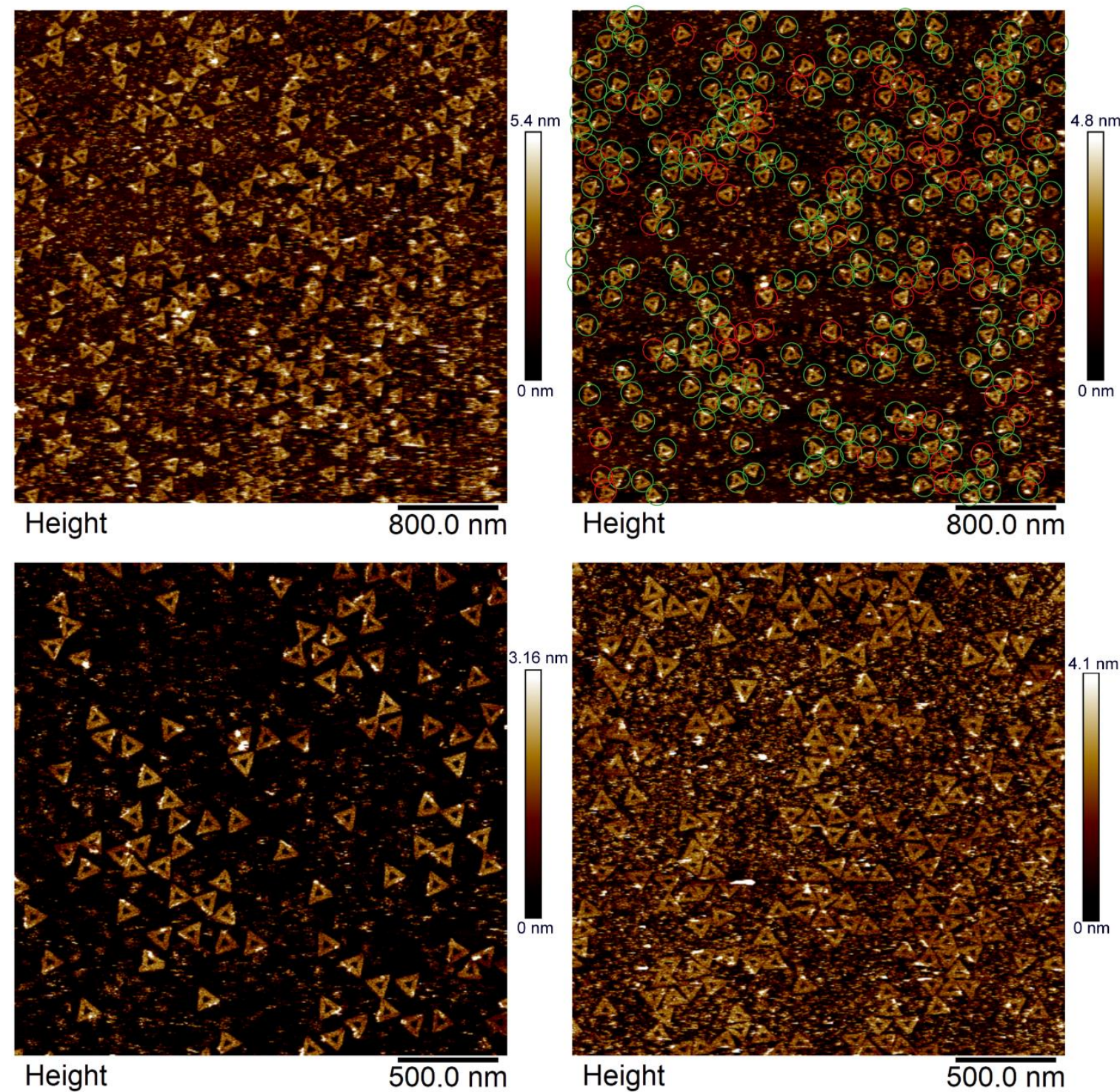

Figure S7. AFM image of triangle origami with one-quarter path points for RNA binding. Green circles refer to correct RNA binding, red circles refer to defective or no RNA binding. 

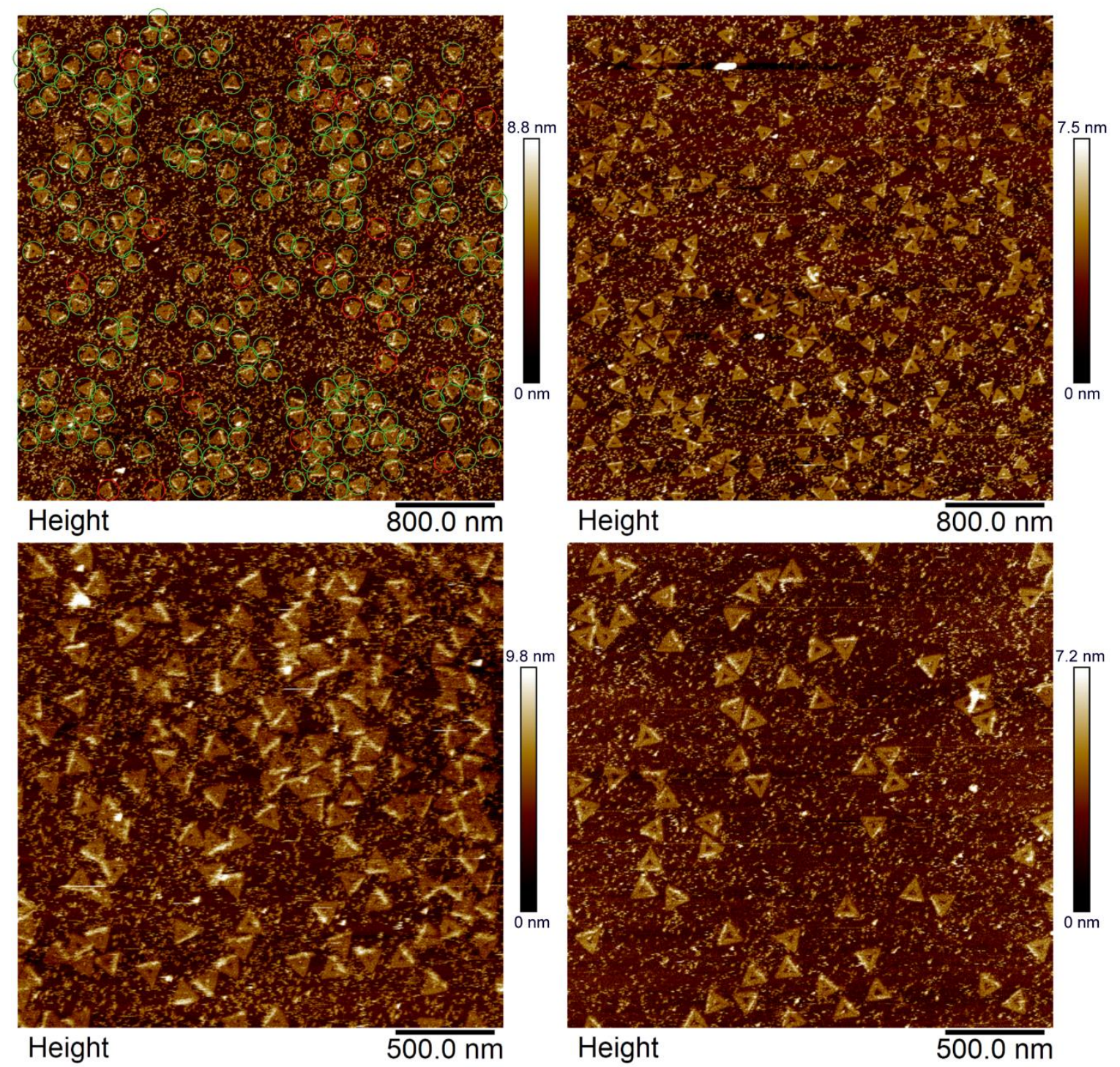

Figure S8. AFM image of triangle origami with half path points for RNA binding. Green circles refer to correct RNA binding, red circles refer to defective or no RNA binding. 

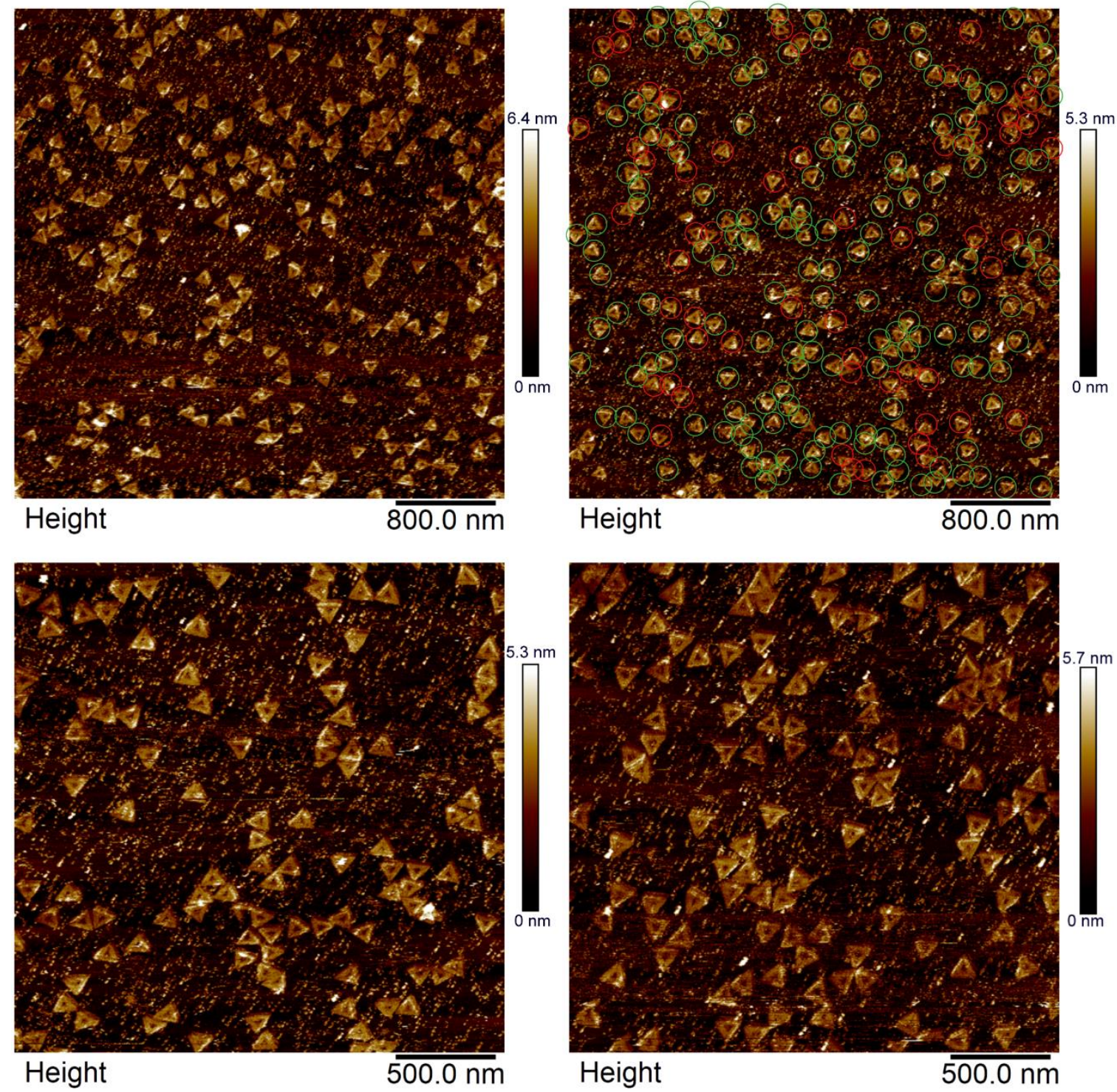

Figure S9. AFM image of triangle origami with three-quarter path points for RNA binding. Green circles refer to correct RNA binding, red circles refer to defective or no RNA binding. 

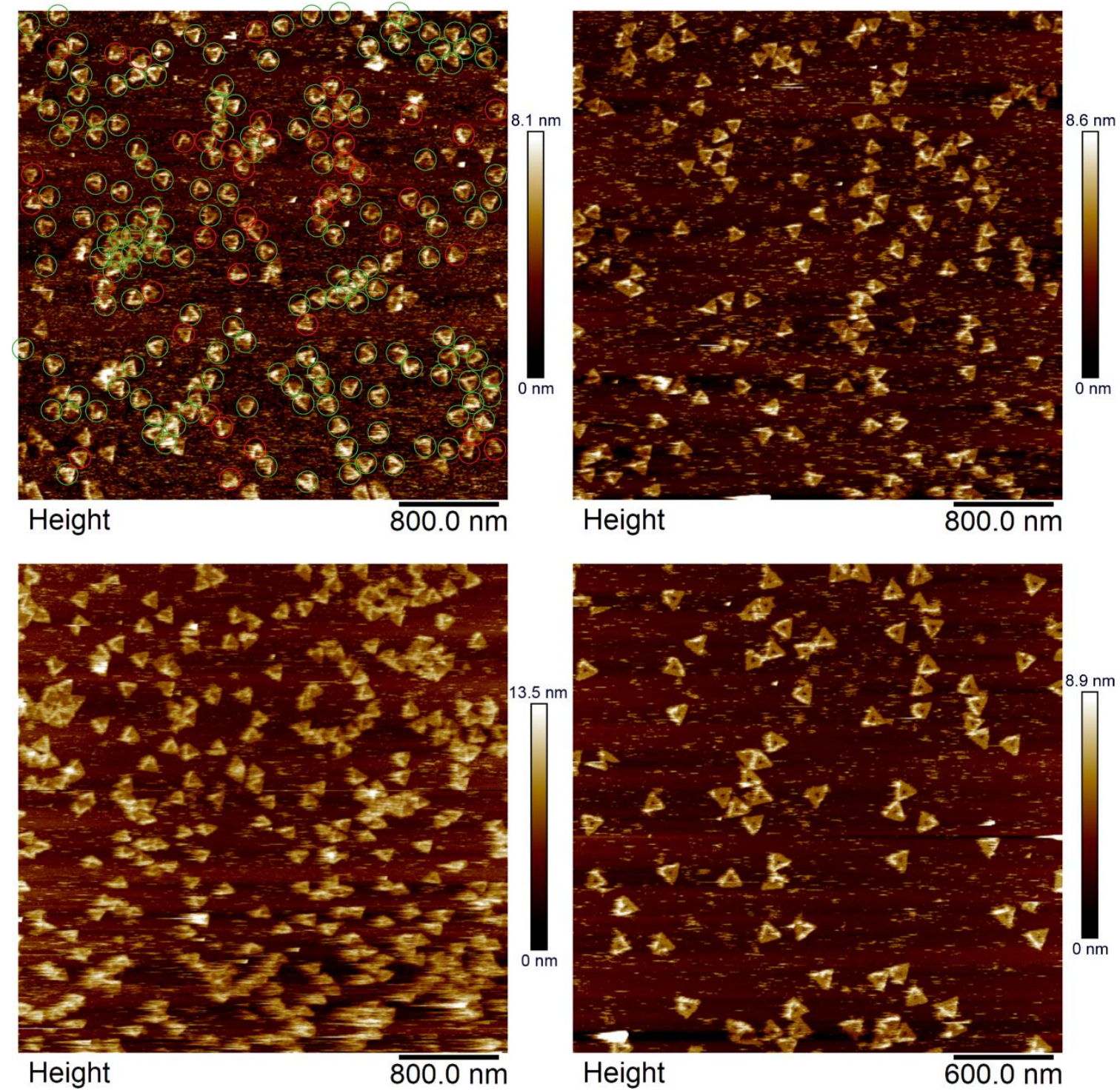

Figure S10. AFM image of triangle origami with all path points for RNA binding. Green circles refer to correct RNA binding, red circles refer to defective or no RNA binding. 

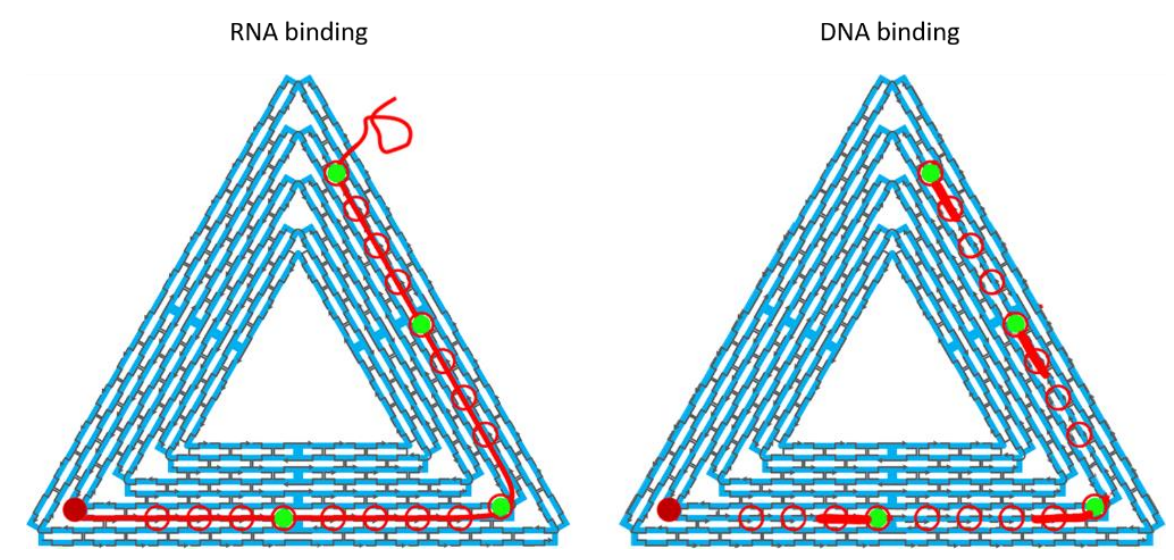

Figure S11. The schematic of RNA binding (left) and the usage of four mimicking DNA strands (right), of which each can bind to one dye-quencher path point and an adjacent path point. The green dots refer to the dye-quencher path points.

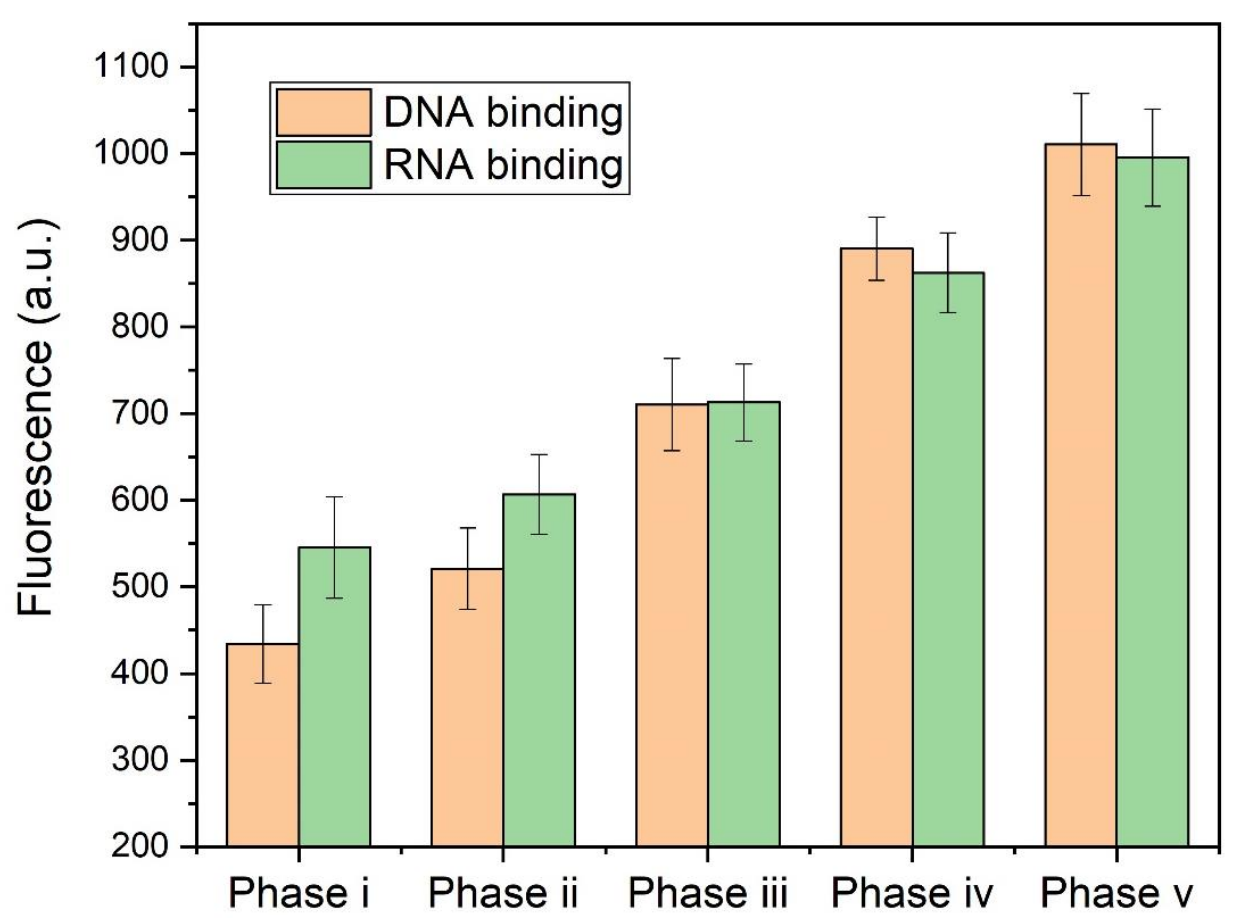

Figure S12. Comparison of fluorescence test using RNA and mimicking DNA. For mimicking DNA, phase $\mathrm{i}$ to $\mathrm{v}$ refer to the DNA-DNA binding on four, three, two, one, and zero dye-quencher path points, respectively, depending on the addition of releasing strands. 


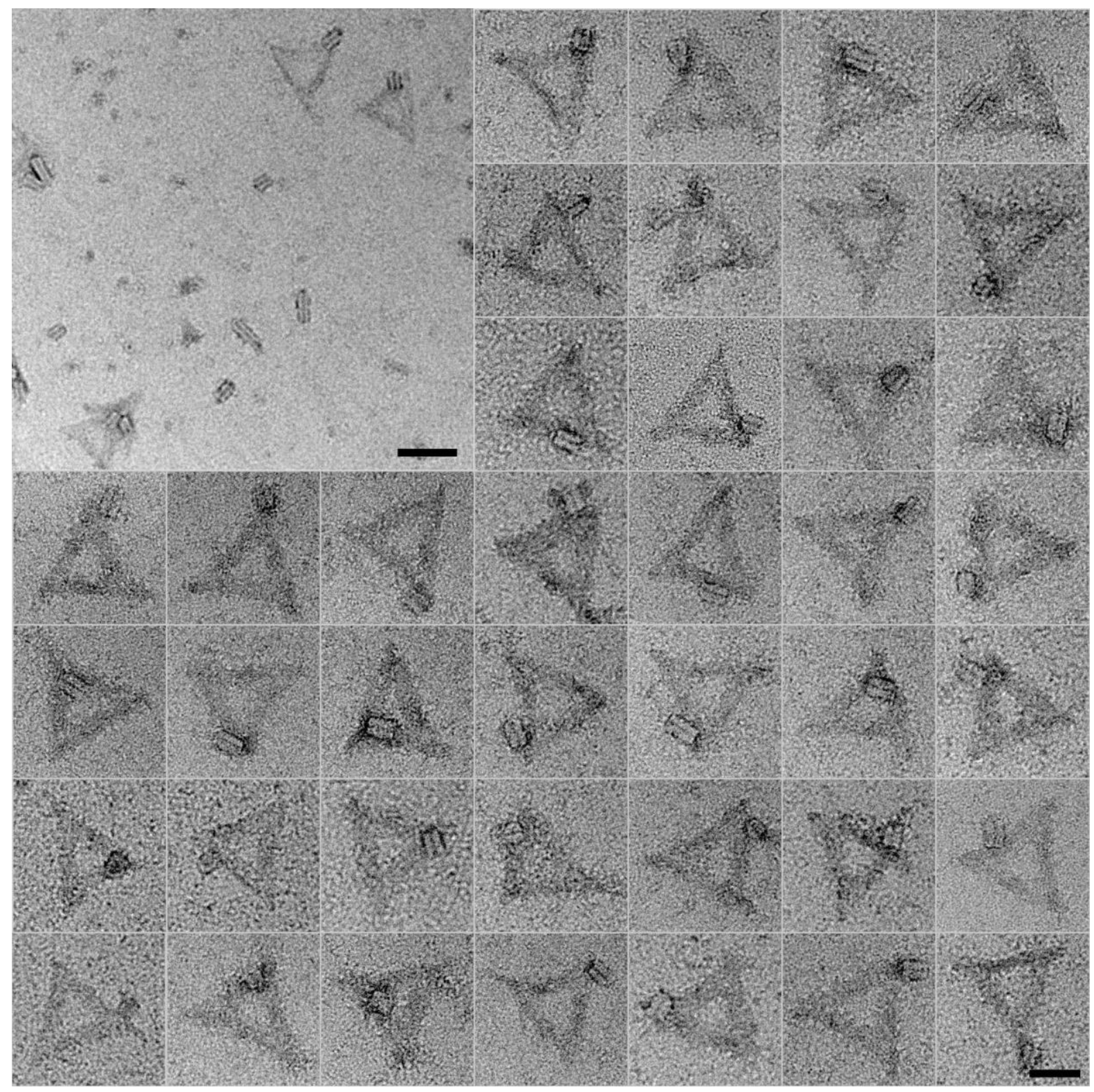

Figure S13. The representative TEM images of triangular origami-protein complex in Phase i. Scale bar; $50 \mathrm{~nm}$ in the zoomed-in TEM image and $100 \mathrm{~nm}$ in the zoomed-out image. Assembly yield was calculated as the ratio between the number of DNA origami with TMV protein in designed pattern (correct position and protein length) and the total number of blank DNA origami, correct DNA origami-protein complex, and failed DNA origami-protein complex with wrong TMV position, length, or aggregates. The total number of counted nanoparticles (NPs) are 87 and the yield of correct DNA origami-protein, failed DNA origami-protein, and blank DNA origami are 37\%, 49\%, 14\%, respectively. 


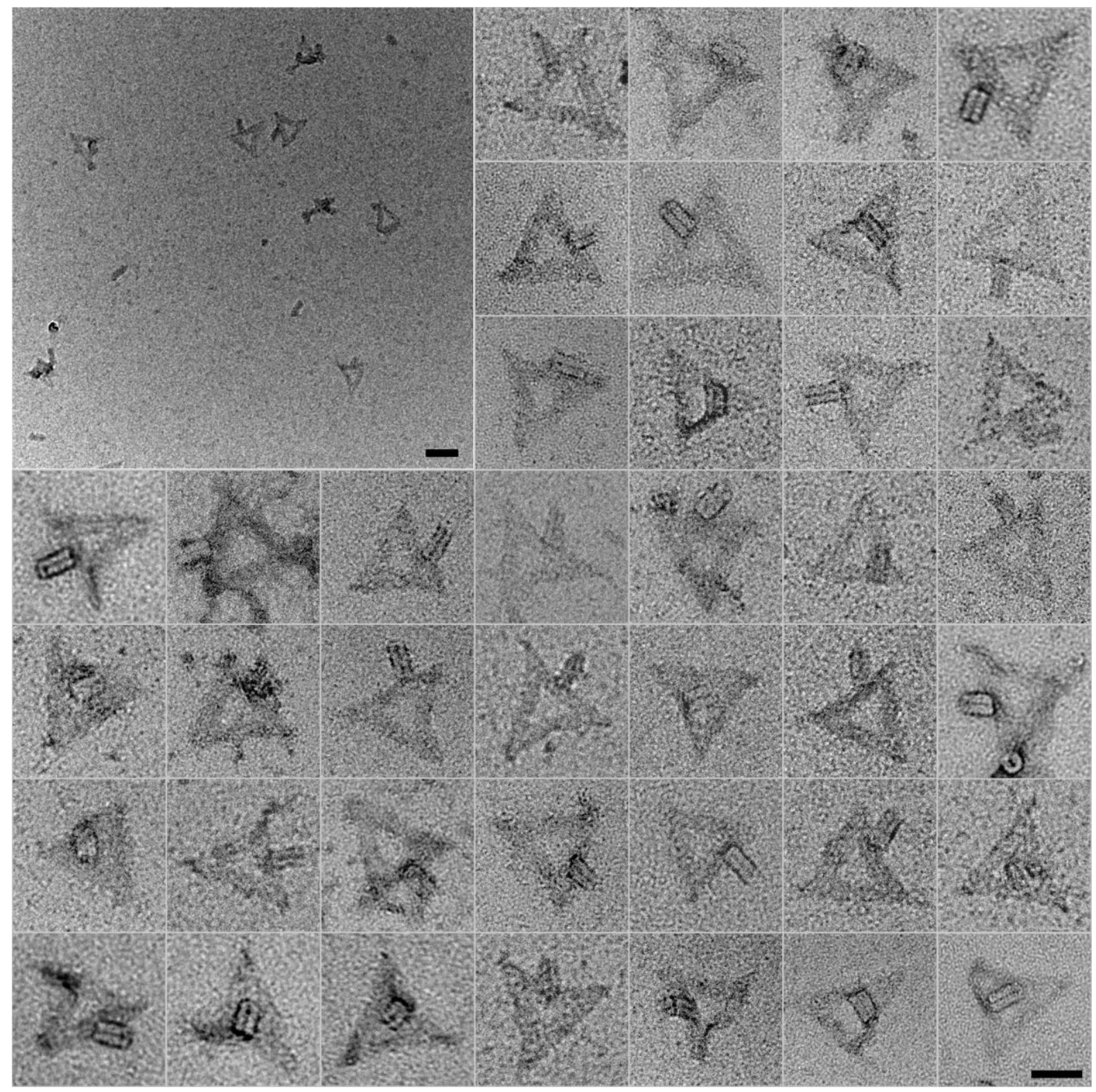

Figure S14. The representative TEM images of triangular origami-protein complex in Phase ii. Scale bar; $50 \mathrm{~nm}$ in the zoomed-in TEM image and $100 \mathrm{~nm}$ in the zoomed-out image. The total number of counted NPs are 83 and the yield of correct DNA origami-protein, failed DNA origami-protein, and blank DNA origami are 38\%, 49\%, 13\%, respectively. 


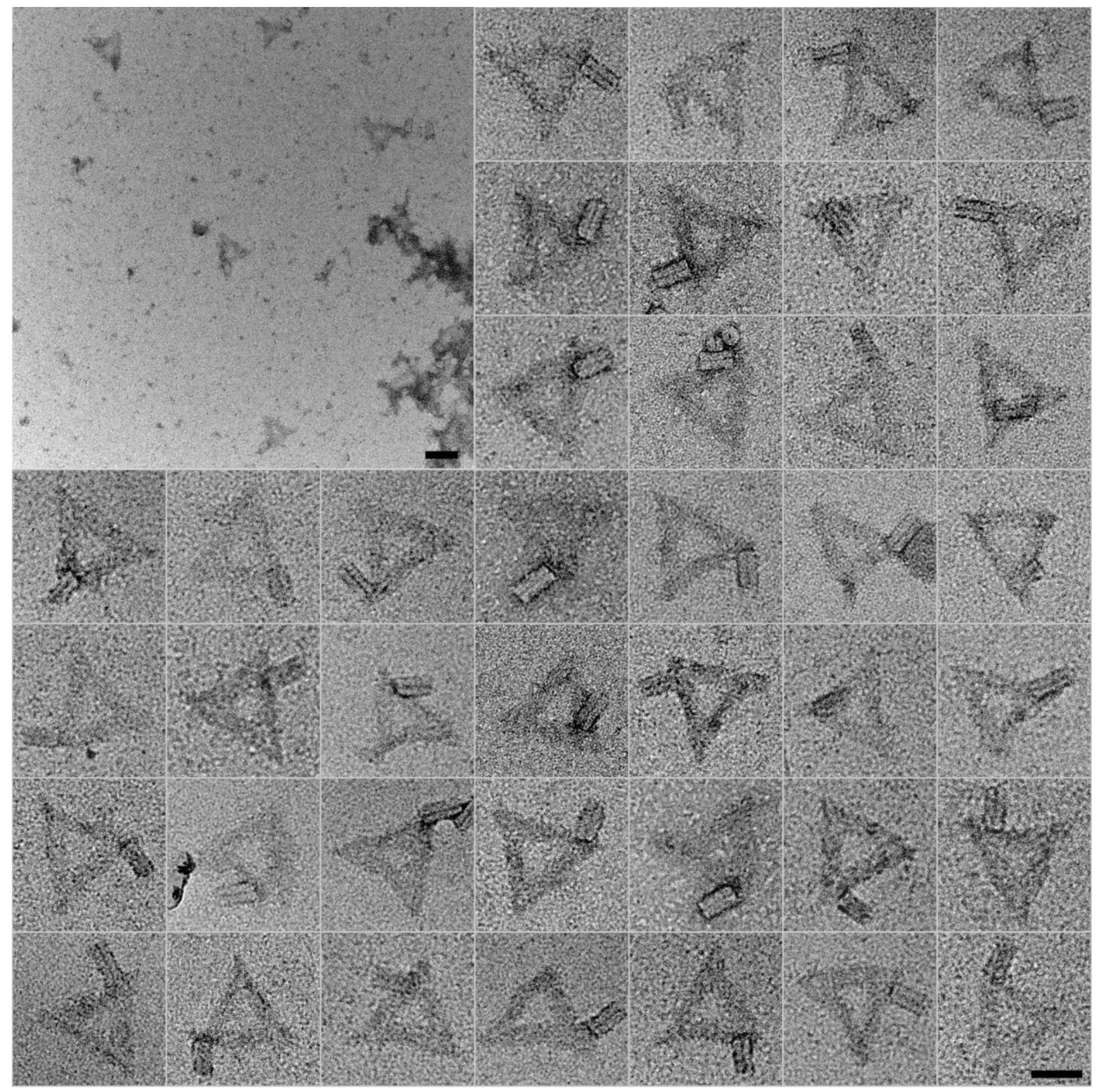

Figure S15. The representative TEM images of triangular origami-protein complex in Phase iii. Scale bar; $50 \mathrm{~nm}$ in the zoomed-in TEM image and $100 \mathrm{~nm}$ in the zoomed-out image. The total number of counted NPs are 102 and the yield of correct DNA origami-protein, failed DNA origami-protein, and blank DNA origami are 33\%, 41\%, 26\%, respectively. 


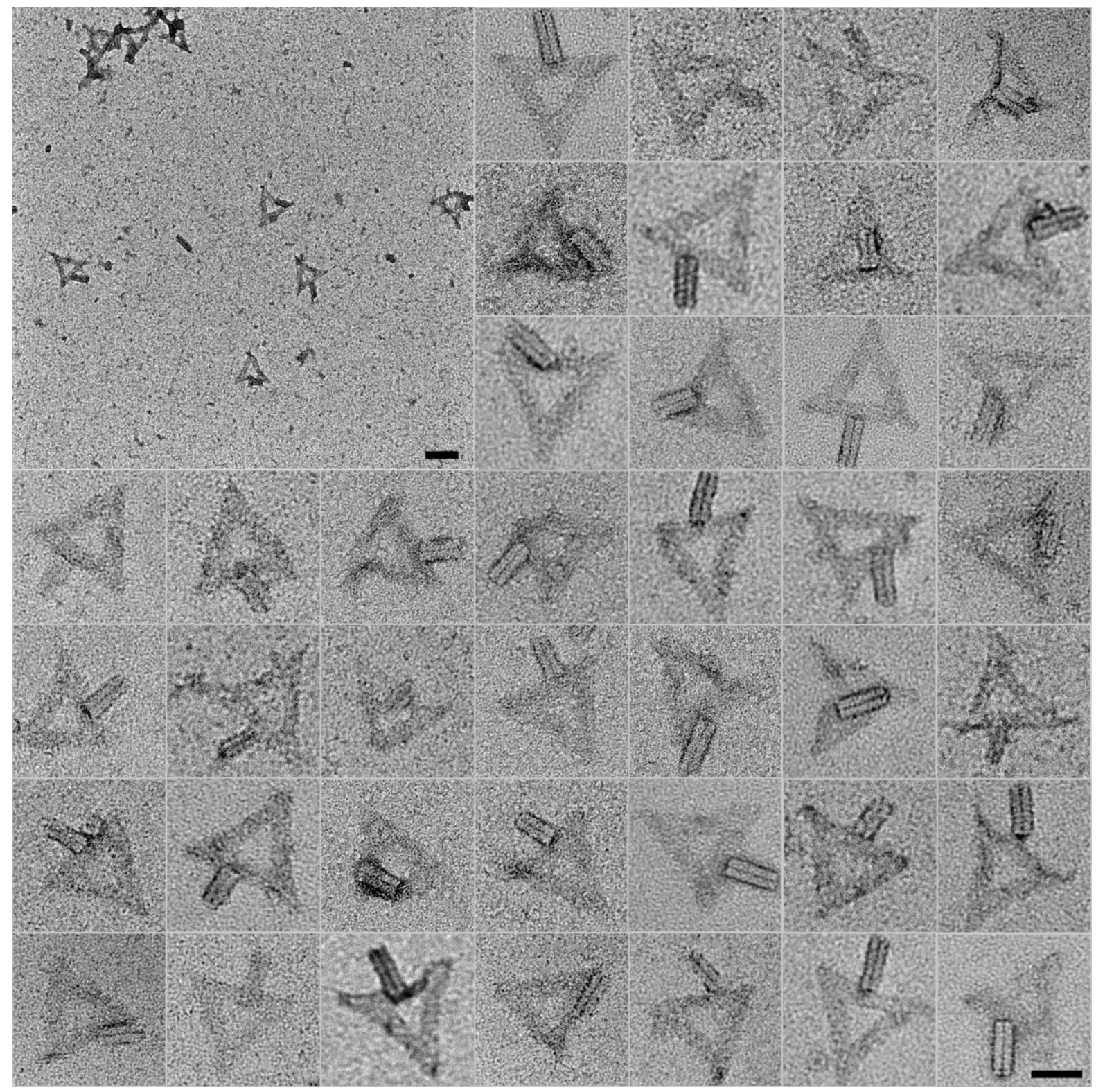

Figure S16. The representative TEM images of triangular origami-protein complex in Phase iv. Scale bar; $50 \mathrm{~nm}$ in the zoomed-in TEM image and $100 \mathrm{~nm}$ in the zoomed-out image. The total number of counted NPs are 94 and the yield of correct DNA origami-protein, failed DNA origami-protein, and blank DNA origami are 31\%, 34\%, 35\%, respectively. 


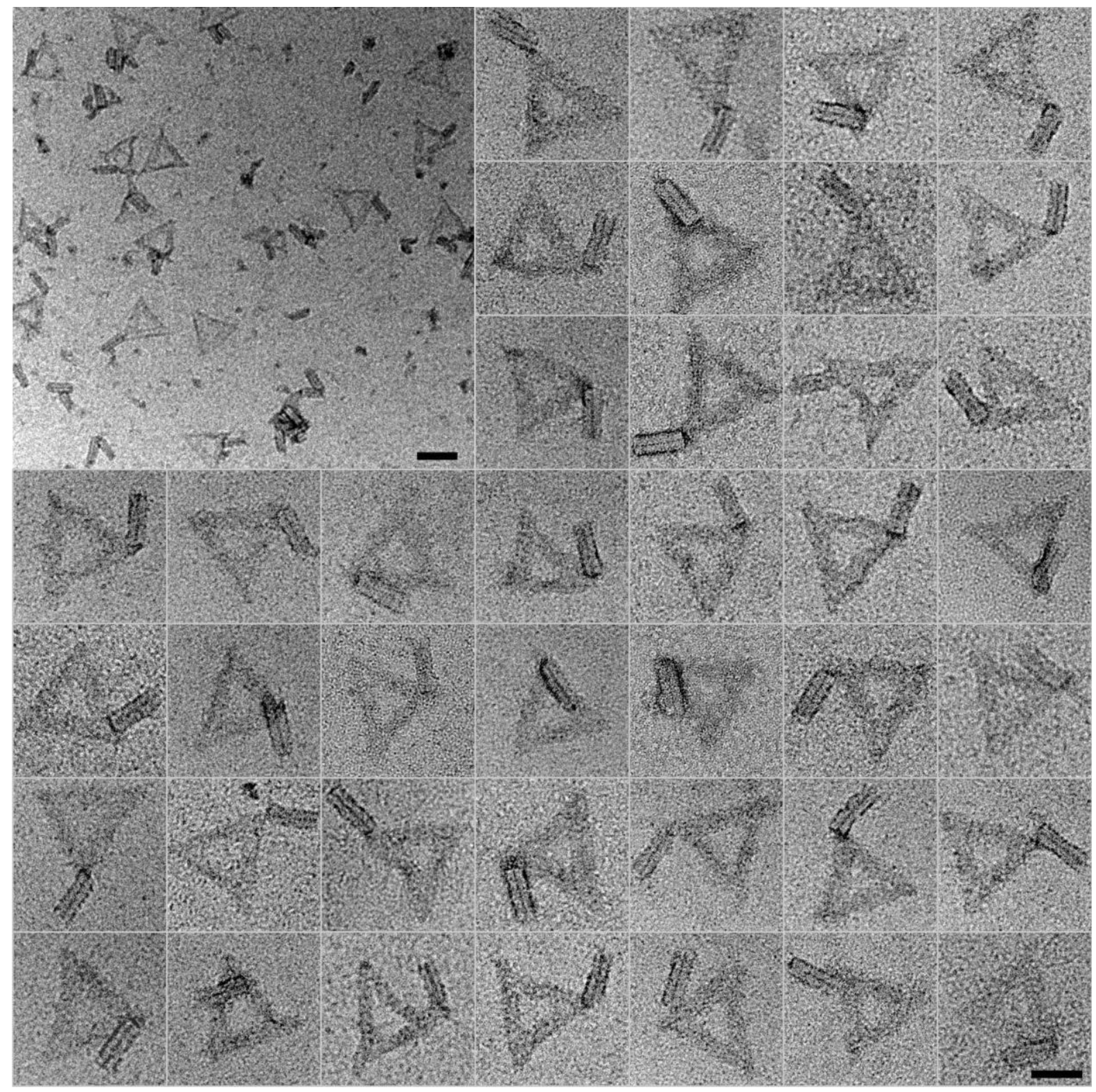

Figure S17. The representative TEM images of triangular origami-protein complex in Phase v. Scale bar; $50 \mathrm{~nm}$ in the zoomed-in TEM image and $100 \mathrm{~nm}$ in the zoomed-out image. The total number of counted NPs are 126 and the yield of correct DNA origami-protein, failed DNA origami-protein, and blank DNA origami are 39\%, 28\%, 33\%, respectively. 


\begin{tabular}{|l|l|l|}
\hline Stage & $\begin{array}{l}\text { Accessible RNA } \\
\text { length }(\mathbf{n t})\end{array}$ & $\begin{array}{l}\text { Expected protein } \\
\text { length }(\mathbf{n m})\end{array}$ \\
\hline Phage i & 563 & 27 \\
\hline Phage ii & 691 & 33 \\
\hline Phage iii & 847 & 40 \\
\hline Phage iv & 1007 & 47 \\
\hline Phage v & 1154 & 54 \\
\hline
\end{tabular}

Figure S18. Characteristics of the valid length of RNA for encapsidation and the corresponding protein length prediction at different phases from $i$ to $v$ during the routing assembly process. Theoretical prediction of the protein length is based on the TMV assembly mechanism, in which one subunit combined with three RNA nucleotides. Note that the last 80-nt sequence for hybridization is not counted for length predication. 


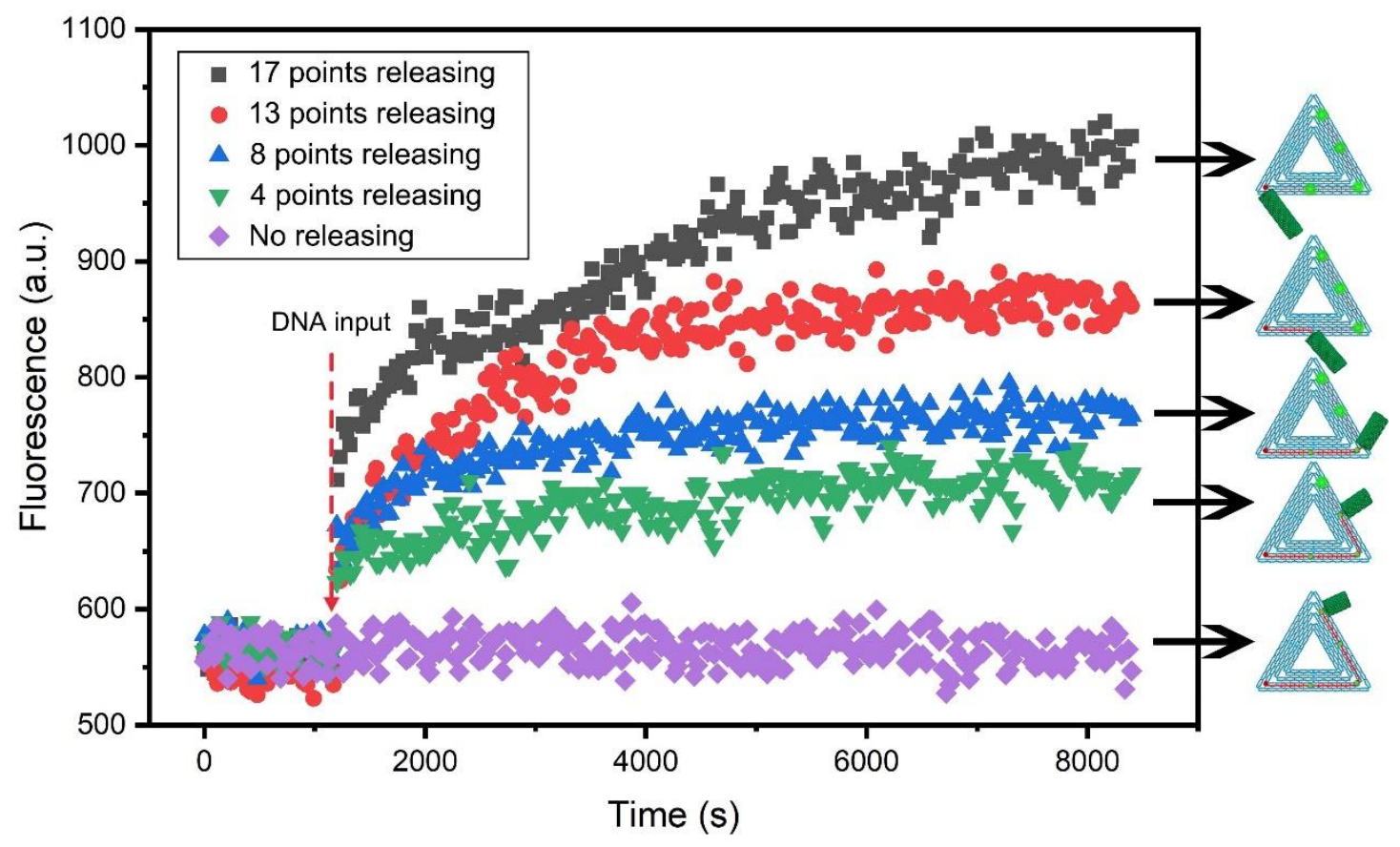

Figure S19. RNA release kinetics monitored by fluorescence over time after the addition of first 4 (green), first 8 (blue), first 13 (red), and all 17 (black) release strands. 
A

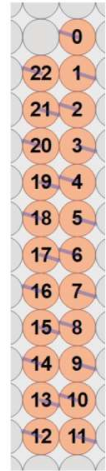

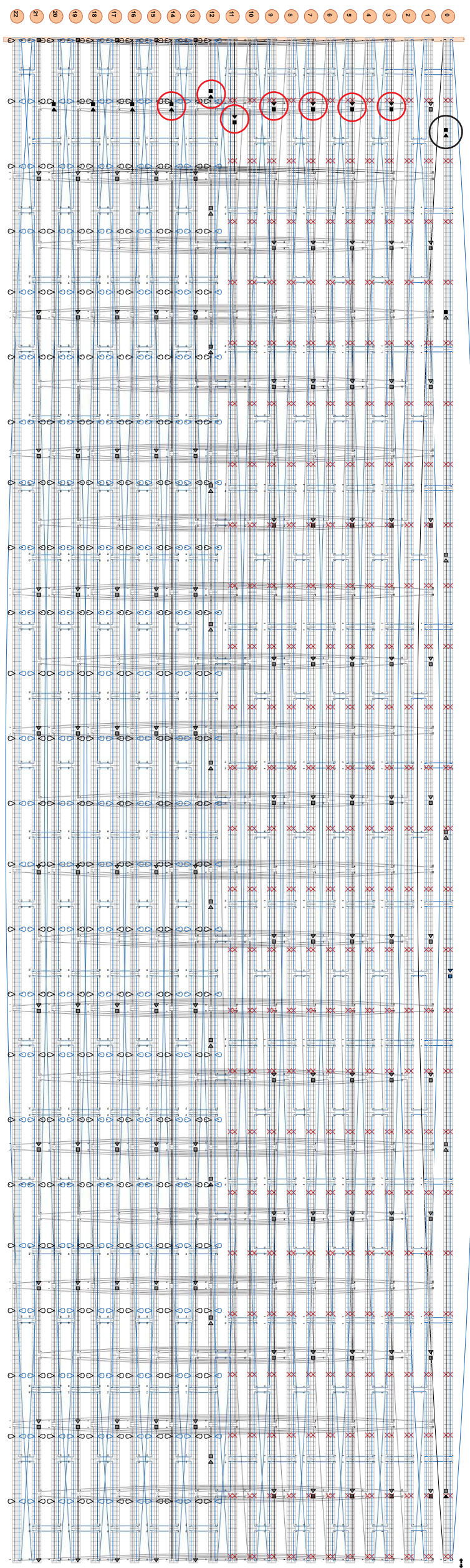

B

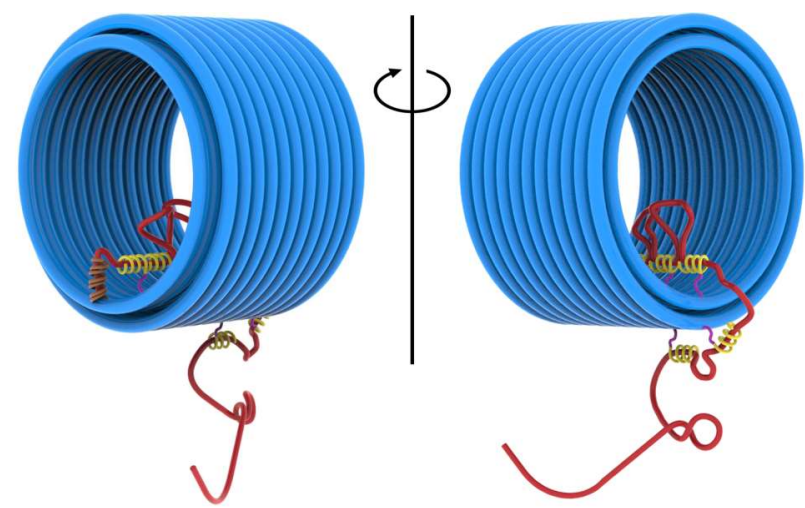

Figure S20. (A) Strand diagram of $23 \mathrm{HB}$ barrel DNA origami design and the pattern design of RNA route. The black circle indicates the anchor point for capturing the 3 '-terminus of RNA and red circles indicate the path points. (B) Model of the design for routing a RNA strand into the $23 \mathrm{HB}$ barrel DNA origami. 


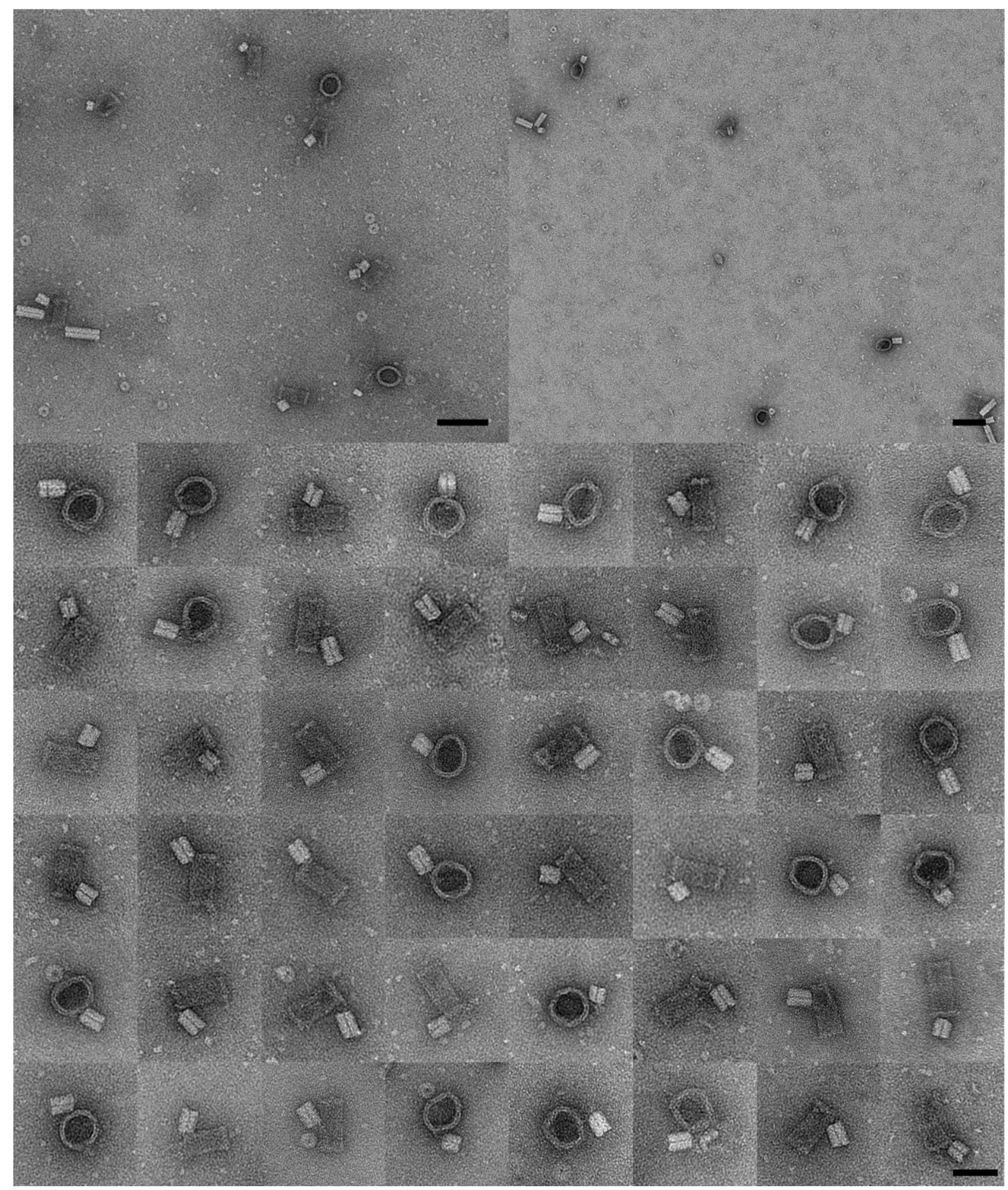

Figure S21. The representative TEM images of protein assembly after the routing process of RNA showing a short protein rod binding to the DNA barrel. Scale bar; $50 \mathrm{~nm}$ in the zoomed-in TEM image and $100 \mathrm{~nm}$ in the zoomed-out image. 

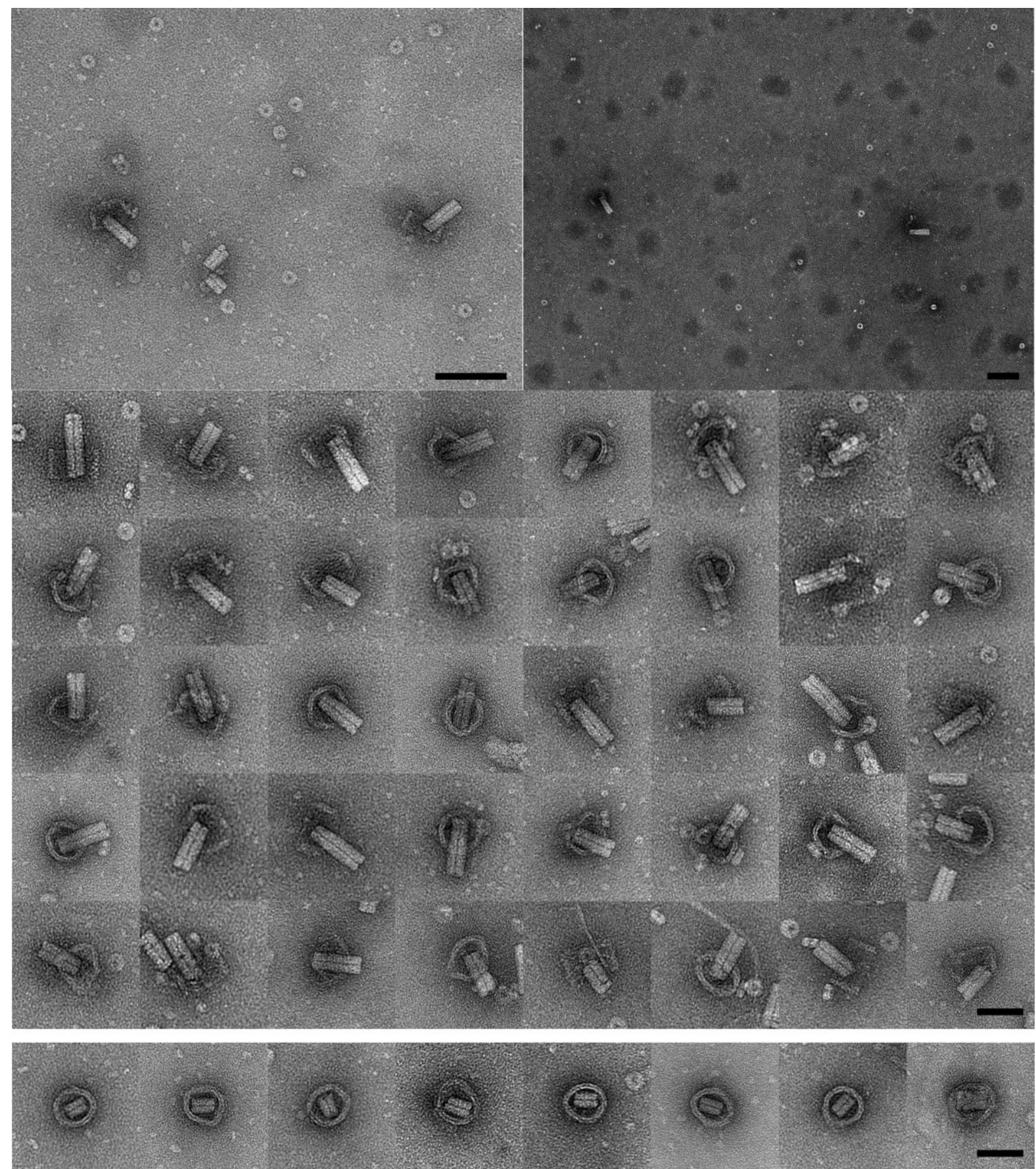

Figure S22. The representative TEM images of the induced growth of protein rod threading the DNA barrel. The bottom images show some protein rods stuck in the DNA barrel. Scale bar; $50 \mathrm{~nm}$ in the zoomed-in TEM image and $100 \mathrm{~nm}$ in the zoomed-out image. 


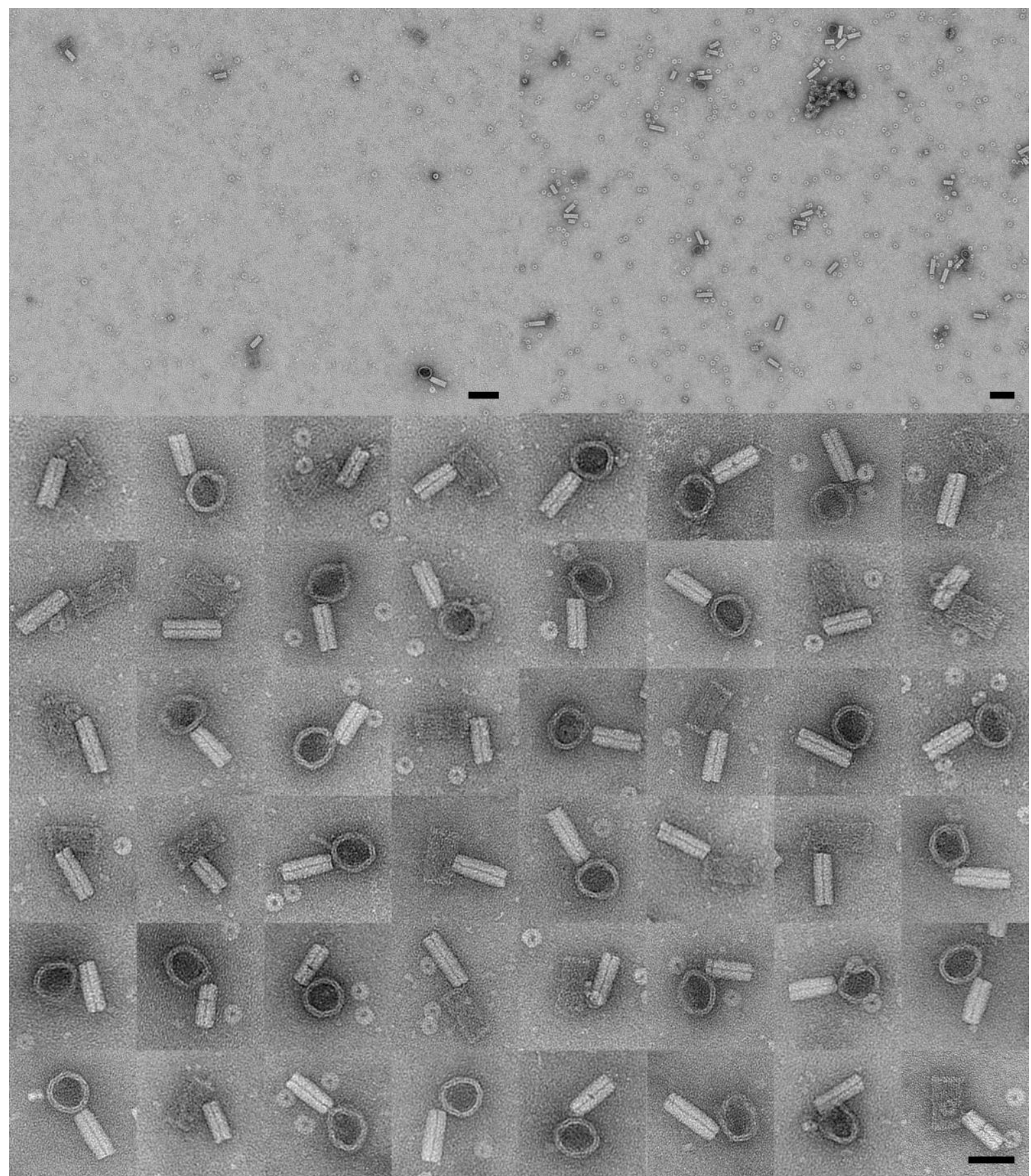

Figure S23. The representative TEM images of protein rod assembled on the outside of DNA barrel without the RNA information routing process. Scale bar; $50 \mathrm{~nm}$ in the zoomed-in TEM image and $100 \mathrm{~nm}$ in the zoomed-out image. 
Sequence of staple strands used in the assembly of the triangular DNA origami

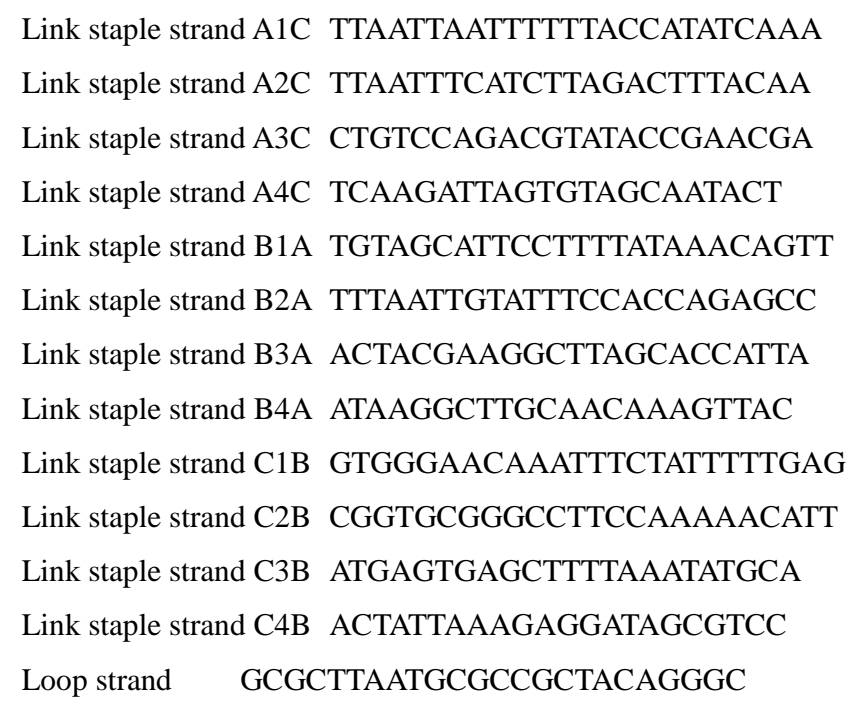

Core staple strand A1 CGGGGTTTCCTCAAGAGAAGGATTTTGAATTA

Core staple strand A2 AGCGTCATGTCTCTGAATTTACCGACTACCTT

Core staple strand A3 TTCATAATCCCCTTATTAGCGTTTTTCTTACC

Core staple strand A4 ATGGTTTATGTCACAATCAATAGATATTAAAC

Core staple strand A5 TTTGATGATTAAGAGGCTGAGACTTGCTCAGTACCAGGCG

Core staple strand A6 CCGGAACCCAGAATGGAAAGCGCAACATGGCT

Core staple strand A7 AAAGACAACATTTTCGGTCATAGCCAAAATCA

Core staple strand A8 GACGGGAGAATTAACTCGGAATAAGTTTATTTCCAGCGCC

Core staple strand A9 GATAAGTGCCGTCGAGCTGAAACATGAAAGTATACAGGAG

Core staple strand A10 TGTACTGGAAATCCTCATTAAAGCAGAGCCAC

Core staple strand A11 CACCGGAAAGCGCGTTTTCATCGGAAGGGCGA

Core staple strand A12 CATTCAACAAACGCAAAGACACCAGAACACCCTGAACAAA

Core staple strand A13 TTTAACGGTTCGGAACCTATTATTAGGGTTGATATAAGTA

Core staple strand A14 CTCAGAGCATATTCACAAACAAATTAATAAGT

Core staple strand A15 GGAGGGAATTTAGCGTCAGACTGTCCGCCTCC

Core staple strand A16 GTCAGAGGGTAATTGATGGCAACATATAAAAGCGATTGAG

Core staple strand A17 TAGCCCGGAATAGGTGAATGCCCCCTGCCTATGGTCAGTG

Core staple strand A18 CCTTGAGTCAGACGATTGGCCTTGCGCCACCC

Core staple strand A19 TCAGAACCCAGAATCAAGTTTGCCGGTAAATA

Core staple strand A20 TTGACGGAAATACATACATAAAGGGCGCTAATATCAGAGA

Core staple strand A21 CAGAGCCAGGAGGTTGAGGCAGGTAACAGTGCCCG

Core staple strand A22 ATTAAAGGCCGTAATCAGTAGCGAGCCACCCT

Core staple strand A23 GATAACCCACAAGAATGTTAGCAAACGTAGAAAATTATTC

Core staple strand A24 GCCGCCAGCATTGACACCACCCTC

Core staple strand A25 AGAGCCGCACCATCGATAGCAGCATGAATTAT

Core staple strand A26 CACCGTCACCTTATTACGCAGTATTGAGTTAAGCCCAATA

Core staple strand A27 AGCCATTTAAACGTCACCAATGAACACCAGAACCA

Core staple strand A28 ATAAGAGCAAGAAACATGGCATGATTAAGACTCCGACTTG

Core staple strand A29 CCATTAGCAAGGCCGGGGGAATTA 
Core staple strand A30 GAGCCAGCGAATACCCAAAAGAACATGAAATAGCAATAGC Core staple strand A31 TATCTTACCGAAGCCCAAACGCAATAATAACGAAAATCACCAG Core staple strand A32 CAGAAGGAAACCGAGGTTTTTAAGAAAAGTAAGCAGATAGCCG Core staple strand A33 CCTTTTTTCATTTAACAATTTCATAGGATTAG Core staple strand A34 TTTAACCTATCATAGGTCTGAGAGTTCCAGTA Core staple strand A35 AGTATAAAATATGCGTTATACAAAGCCATCTT Core staple strand A36 CAAGTACCTCATTCCAAGAACGGGAAATTCAT Core staple strand A37 AGAGAATAACATAAAAACAGGGAAGCGCATTA Core staple strand A38 AAAACAAAATTAATTAAATGGAAACAGTACATTAGTGAAT Core staple strand A39 TTATCAAACCGGCTTAGGTTGGGTAAGCCTGT Core staple strand A40 TTAGTATCGCCAACGCTCAACAGTCGGCTGTC Core staple strand A41 TTTCCTTAGCACTCATCGAGAACAATAGCAGCCTTTACAG Core staple strand A42 AGAGTCAAAAATCAATATATGTGATGAAACAAACATCAAG Core staple strand A43 ACTAGAAATATATAACTATATGTACGCTGAGA Core staple strand A44 TCAATAATAGGGCTTAATTGAGAATCATAATT Core staple strand A45 AACGTCAAAAATGAAAAGCAAGCCGTTTTTATGAAACCAA Core staple strand A46 GAGCAAAAGAAGATGAGTGAATAACCTTGCTTATAGCTTA Core staple strand A47 GATTAAGAAATGCTGATGCAAATCAGAATAAA Core staple strand A48 CACCGGAATCGCCATATTTAACAAAATTTACG Core staple strand A49 AGCATGTATTTCATCGTAGGAATCAAACGATTTTTTGTTT Core staple strand A50 ACATAGCGCTGTAAATCGTCGCTATTCATTTCAATTACCT Core staple strand A51 GTTAAATACAATCGCAAGACAAAGCCTTGAAA Core staple strand A52 CCCATCCTCGCCAACATGTAATTTAATAAGGC Core staple strand A53 TCCCAATCCAAATAAGATTACCGCGCCCAATAAATAATAT Core staple strand A54 TCCCTTAGAATAACGCGAGAAAACTTTTACCGACC Core staple strand A55 GTGTGATAAGGCAGAGGCATTTTCAGTCCTGA Core staple strand A56 ACAAGAAAGCAAGCAAATCAGATAACAGCCATATTATTTA Core staple strand A57 GTTTGAAATTCAAATATATTTTAG Core staple strand A58 AATAGATAGAGCCAGTAATAAGAGATTTAATG Core staple strand A59 GCCAGTTACAAAATAATAGAAGGCTTATCCGGTTATCAAC Core staple strand A60 TTCTGACCTAAAATATAAAGTACCGACTGCAGAAC Core staple strand A61 GCGCCTGTTATTCTAAGAACGCGATTCCAGAGCCTAATTT Core staple strand A62 TCAGCTAAAAAAGGTAAAGTAATT Core staple strand A63 ACGCTAACGAGCGTCTGGCGTTTTAGCGAACCCAACATGT Core staple strand A64 ACGACAATAAATCCCGACTTGCGGGAGATCCTGAATCTTACCA Core staple strand A65 TGCTATTTTGCACCCAGCTACAATTTTGTTTTGAAGCCTTAAA Core staple strand B1 TCATATGTGTAATCGTAAAACTAGTCATTTTC Core staple strand B2 GTGAGAAAATGTGTAGGTAAAGATACAACTTT Core staple strand B3 GGCATCAAATTTGGGGCGCGAGCTAGTTAAAG Core staple strand B4 TTCGAGCTAAGACTTCAAATATCGGGAACGAG Core staple strand B5 ACAGTCAAAGAGAATCGATGAACGACCCCGGTTGATAATC Core staple strand B6 ATAGTAGTATGCAATGCCTGAGTAGGCCGGAG Core staple strand B7 AACCAGACGTTTAGCTATATTTTCTTCTACTA Core staple strand B8 GAATACCACATTCAACTTAAGAGGAAGCCCGATCAAAGCG 
Core staple strand B9 AGAAAAGCCCCAAAAAGAGTCTGGAGCAAACAATCACCAT Core staple strand B10 CAATATGACCCTCATATATTTTAAAGCATTAA Core staple strand B11 CATCCAATAAATGGTCAATAACCTCGGAAGCA Core staple strand B12 AACTCCAAGATTGCATCAAAAAGATAATGCAGATACATAA Core staple strand B13 CGTTCTAGTCAGGTCATTGCCTGACAGGAAGATTGTATAA Core staple strand B14 CAGGCAAGATAAAAATTTTTAGAATATTCAAC Core staple strand B15 GATTAGAGATTAGATACATTTCGCAAATCATA Core staple strand B16 CGCCAAAAGGAATTACAGTCAGAAGCAAAGCGCAGGTCAG Core staple strand B17 GCAAATATTTAAATTGAGATCTACAAAGGCTACTGATAAA Core staple strand B18 TTAATGCCTTATTTCAACGCAAGGGCAAAGAA Core staple strand B19 TTAGCAAATAGATTTAGTTTGACCAGTACCTT Core staple strand B20 TAATTGCTTTACCCTGACTATTATGAGGCATAGTAAGAGC Core staple strand B21 ATAAAGCCTTTGCGGGAGAAGCCTGGAGAGGGTAG Core staple strand B22 TAAGAGGTCAATTCTGCGAACGAGATTAAGCA Core staple strand B23 AACACTATCATAACCCATCAAAAATCAGGTCTCCTTTTGA Core staple strand B24 ATGACCCTGTAATACTTCAGAGCA Core staple strand B25 TAAAGCTATATAACAGTTGATTCCCATTTTTG Core staple strand B26 CGGATGGCACGAGAATGACCATAATCGTTTACCAGACGAC Core staple strand B27 TAATTGCTTGGAAGTTTCATTCCAAATCGGTTGTA Core staple strand B28 GATAAAAACCAAAATATTAAACAGTTCAGAAATTAGAGCT Core staple strand B29 ACTAAAGTACGGTGTCGAATATAA Core staple strand B30 TGCTGTAGATCCCCCTCAAATGCTGCGAGAGGCTTTTGCA Core staple strand B31 AAAGAAGTTTTGCCAGCATAAATATTCATTGACTCAACATGTT Core staple strand B32 AATACTGCGGAATCGTAGGGGGTAATAGTAAAATGTTTAGACT Core staple strand B33 AGGGATAGCTCAGAGCCACCACCCCATGTCAA Core staple strand B34 CAACAGTTTATGGGATTTTGCTAATCAAAAGG Core staple strand B35 GCCGCTTTGCTGAGGCTTGCAGGGGAAAAGGT Core staple strand B36 GCGCAGACTCCATGTTACTTAGCCCGTTTTAA Core staple strand B37 ACAGGTAGAAAGATTCATCAGTTGAGATTTAG Core staple strand B38 CCTCAGAACCGCCACCCAAGCCCAATAGGAACGTAAATGA Core staple strand B39 ATTTTCTGTCAGCGGAGTGAGAATACCGATAT Core staple strand B40 ATTCGGTCTGCGGGATCGTCACCCGAAATCCG Core staple strand B41 CGACCTGCGGTCAATCATAAGGGAACGGAACAACATTATT Core staple strand B42 AGACGTTACCATGTACCGTAACACCCCTCAGAACCGCCAC Core staple strand B43 CACGCATAAGAAAGGAACAACTAAGTCTTTCC Core staple strand B44 ATTGTGTCTCAGCAGCGAAAGACACCATCGCC Core staple strand B45 TTAATAAAACGAACTAACCGAACTGACCAACTCCTGATAA Core staple strand B46 AGGTTTAGTACCGCCATGAGTTTCGTCACCAGGATCTAAA Core staple strand B47 GTTTTGTCAGGAATTGCGAATAATCCGACAAT Core staple strand B48 GACAACAAGCATCGGAACGAGGGTGAGATTTG Core staple strand B49 TATCATCGTTGAAAGAGGACAGATGGAAGAAAAATCTACG Core staple strand B50 AGCGTAACTACAAACTACAACGCCTATCACCGTACTCAGG Core staple strand B51 TAGTTGCGAATTTTTTCACGTTGATCATAGTT Core staple strand B52 GTACAACGAGCAACGGCTACAGAGGATACCGA 
Core staple strand B53 ACCAGTCAGGACGTTGGAACGGTGTACAGACCGAAACAAA Core staple strand B54 ACAGACAGCCCAAATCTCCAAAAAAAAATTTCTTA Core staple strand B55 AACAGCTTGCTTTGAGGACTAAAGCGATTATA Core staple strand B56 CCAAGCGCAGGCGCATAGGCTGGCAGAACTGGCTCATTAT Core staple strand B57 CGAGGTGAGGCTCCAAAAGGAGCC Core staple strand B58 ACCCCCAGACTTTTTCATGAGGAACTTGCTTT Core staple strand B59 ACCTTATGCGATTTTATGACCTTCATCAAGAGCATCTTTG Core staple strand B60 CGGTTTATCAGGTTTCCATTAAACGGGAATACACT Core staple strand B61 AAAACACTTAATCTTGACAAGAACTTAATCATTGTGAATT Core staple strand B62 GGCAAAAGTAAAATACGTAATGCC Core staple strand B63 TGGTTTAATTTCAACTCGGATATTCATTACCCACGAAAGA Core staple strand B64 ACCAACCTAAAAAATCAACGTAACAAATAAATTGGGCTTGAGA Core staple strand B65 CCTGACGAGAAACACCAGAACGAGTAGGCTGCTCATTCAGTGA Core staple strand C1 TCGGGAGATATACAGTAACAGTACAAATAATT Core staple strand C2 CCTGATTAAAGGAGCGGAATTATCTCGGCCTC Core staple strand C3 GCAAATCACCTCAATCAATATCTGCAGGTCGA Core staple strand C4 CGACCAGTACATTGGCAGATTCACCTGATTGC Core staple strand C5 TGGCAATTTTTAACGTCAGATGAAAACAATAACGGATTCG Core staple strand C6 AAGGAATTACAAAGAAACCACCAGTCAGATGA Core staple strand C7 GGACATTCACCTCAAATATCAAACACAGTTGA Core staple strand C8 TTGACGAGCACGTATACTGAAATGGATTATTTAATAAAAG Core staple strand C9 CCTGATTGCTTTGAATTGCGTAGATTTTCAGGCATCAATA Core staple strand C10 TAATCCTGATTATCATTTTGCGGAGAGGAAGG Core staple strand C11 TTATCTAAAGCATCACCTTGCTGATGGCCAAC Core staple strand C12 AGAGATAGTTTGACGCTCAATCGTACGTGCTTTCCTCGTT Core staple strand C13 GATTATACACAGAAATAAAGAAATACCAAGTTACAAAATC Core staple strand C14 TAGGAGCATAAAAGTTTGAGTAACATTGTTTG Core staple strand C15 TGACCTGACAAATGAAAAATCTAAAATATCTT Core staple strand C16 AGAATCAGAGCGGGAGATGGAAATACCTACATAACCCTTC Core staple strand C17 GCGCAGAGGCGAATTAATTATTTGCACGTAAATTCTGAAT Core staple strand C18 AATGGAAGCGAACGTTATTAATTTCTAACAAC Core staple strand C19 TAATAGATCGCTGAGAGCCAGCAGAAGCGTAA Core staple strand C20 GAATACGTAACAGGAAAAACGCTCCTAAACAGGAGGCCGA Core staple strand C21 TCAATAGATATTAAATCCTTTGCCGGTTAGAACCT Core staple strand C22 CAATATTTGCCTGCAACAGTGCCATAGAGCCG Core staple strand C23 TTAAAGGGATTTTAGATACCGCCAGCCATTGCGGCACAGA Core staple strand C24 ACAATTCGACAACTCGTAATACAT Core staple strand C25 TTGAGGATGGTCAGTATTAACACCTTGAATGG Core staple strand C26 CTATTAGTATATCCAGAACAATATCAGGAACGGTACGCCA Core staple strand C27 CGCGAACTAAAACAGAGGTGAGGCTTAGAAGTATT Core staple strand C28 GAATCCTGAGAAGTGTATCGGCCTTGCTGGTACTTTAATG Core staple strand C29 ACCACCAGCAGAAGATGATAGCCC Core staple strand C30 TAAAACATTAGAAGAACTCAAACTTTTTATAATCAGTGAG Core staple strand C31 GCCACCGAGTAAAAGAACATCACTTGCCTGAGCGCCATTAAAA 
Core staple strand C32 TCTTTGATTAGTAATAGTCTGTCCATCACGCAAATTAACCGTT Core staple strand C33 CGCGTCTGATAGGAACGCCATCAACTTTTACA Core staple strand C34 AGGAAGATGGGGACGACGACAGTAATCATATT Core staple strand C35 CTCTAGAGCAAGCTTGCATGCCTGGTCAGTTG Core staple strand C36 CCTTCACCGTGAGACGGGCAACAGCAGTCACA Core staple strand C37 CGAGAAAGGAAGGGAAGCGTACTATGGTTGCT Core staple strand C38 GCTCATTTTTTAACCAGCCTTCCTGTAGCCAGGCATCTGC Core staple strand C39 CAGTTTGACGCACTCCAGCCAGCTAAACGACG Core staple strand C40 GCCAGTGCGATCCCCGGGTACCGAGTTTTTCT Core staple strand C41 TTTCACCAGCCTGGCCCTGAGAGAAAGCCGGCGAACGTGG Core staple strand C42 GTAACCGTCTTTCATCAACATTAAAATTTTTGTTAAATCA Core staple strand C43 ACGTTGTATTCCGGCACCGCTTCTGGCGCATC Core staple strand C44 CCAGGGTGGCTCGAATTCGTAATCCAGTCACG Core staple strandC45 TAGAGCTTGACGGGGAGTTGCAGCAAGCGGTCATTGGGCG Core staple strand C46 GTTAAAATTCGCATTAATGTGAGCGAGTAACACACGTTGG Core staple strand C47 TGTAGATGGGTGCCGGAAACCAGGAACGCCAG Core staple strand C48 GGTTTTCCATGGTCATAGCTGTTTGAGAGGCG Core staple strand C49 GTTTGCGTCACGCTGGTTTGCCCCAAGGGAGCCCCCGATT Core staple strand C50 GGATAGGTACCCGTCGGATTCTCCTAAACGTTAATATTTT Core staple strand C51 AGTTGGGTCAAAGCGCCATTCGCCCCGTAATG Core staple strand C52 CGCGCGGGCCTGTGTGAAATTGTTGGCGATTA Core staple strand C53 CTAAATCGGAACCCTAAGCAGGCGAAAATCCTTCGGCCAA Core staple strand C54 CGGCGGATTGAATTCAGGCTGCGCAACGGGGGATG Core staple strand C55 TGCTGCAAATCCGCTCACAATTCCCAGCTGCA Core staple strand C56 TTAATGAAGTTTGATGGTGGTTCCGAGGTGCCGTAAAGCA Core staple strand C57 TGGCGAAATGTTGGGAAGGGCGAT Core staple strand C58 TGTCGTGCACACAACATACGAGCCACGCCAGC Core staple strand C59 CAAGTTTTTTGGGGTCGAAATCGGCAAAATCCGGGAAACC Core staple strand C60 TCTTCGCTATTGGAAGCATAAAGTGTATGCCCGCT Core staple strand C61 TTCCAGTCCTTATAAATCAAAAGAGAACCATCACCCAAAT Core staple strand C62 GCGCTCACAAGCCTGGGGTGCCTA Core staple strand C63 CGATGGCCCACTACGTATAGCCCGAGATAGGGATTGCGTT Core staple strand C64 AACTCACATTATTGAGTGTTGTTCCAGAAACCGTCTATCAGGG Core staple strand C65 ACGTGGACTCCAACGTCAAAGGGCGAATTTGGAACAAGAGTCC 
Sequence of capturing strands and routing strands used to replace corresponding handle staple strands in the assembly of the triangular DNA origami (red: 40 nt or 13 nt long extensions of strands for binding RNA; blue: 16 nt long extensions of strands for toehold exchange reaction to release RNA)

Capturing strand A31

TATCTTACCGAAGCCCAAACGCAATAATAACGAAAATCACGACGAATTCGAGCGCACCACGTGTGATTACGGAC ACAATC

Capturing strand B64

TATGGTCGACCTGCAGGCGGCCGCACTAGTGATTGGGAGACAGAACCAACCTAAAAAATCAACGTAACAAAT AAATTGGGCTTGAGA

Routing strand B58

GACTAGAGGTCCAAATAATAATAATAATATTTACCCCCAGACTTTTTCATGAGGAACTTGCTTT

Routing strand B59

ACCTTATGCGATTTTATGACCTTCATCAAGAGCATCTTTGTTTAGCACCCACTATACTTGCTCAAGTTGCAG

Routing strand B52

TCGAAAGAGCTCCAAAAAAAAAAAAAAAATTTGTACAACGAGCAACGGCTACAGAGGATACCGA

Routing strand B53

ACCAGTCAGGACGTTGGAACGGTGTACAGACCGAAACAAATTTCCTACCACGAATTCTAACCAGAAGAGCTC Routing strand B44

TCTGATCAATTCTAGACTCTAATGCAGTCTTTATTGTGTCTCAGCAGCGAAAGACACCATCGCC

Routing strand B45

TTAATAAAACGAACTAACCGAACTGACCAACTCCTGATAATTTTCACCCCTATTGCCAAAAGATCCGGTTCC Routing strand B36

FAM-CGCTCCTTGTGGCAGATATGAGATCCGTATTTACTTAGCCCGTTTTAATTCGAGCTAAGACTTC

Routing strand B4

AAATATCGGGAACGAGGCGCAGACTCCATGTTTTTGATTGAAAAGGGTGACAAATTATTTATCG-BHQ1

Routing strand B7

CTACGAGTAGCATCGCATTCAGGATTCTCTTTAACCAGACGTTTAGCTATATTTTCTTCTACTA

Routing strand B8

GAATACCACATTCAACTTAAGAGGAAGCCCGATCAAAGCGTTTATCTCACCTACATAGGTGCGTCGTCTACT

Routing strand B15

GGGGTTCGCCTGAATACTACGTGGTAAAGTTTGATTAGAGATTAGATACATTTCGCAAATCATA

Routing strand B16

CGCCAAAAGGAATTACAGTCAGAAGCAAAGCGCAGGTCAGTTTGTGTTGGACATCATAATTTCGGCAGTCGT Routing strand B22

TATTTCTAGTGTCCGGACGCTCTCCTGGGTTTTAAGAGGTCAATTCTGCGAACGAGATTAAGCA

Routing strand B23

AACACTATCATAACCCATCAAAAATCAGGTCTCCTTTTGATTTCAGGTGGCACCCAAACACTTCTATTATTC

Routing strand B27

GTGACTAGCGGGTCGGACTAGAGTGGCGATTTTAATTGCTTGGAAGTTTCATTCCAAATCGGTTGTA

Routing strand B28

GATAAAAACCAAAATATTAAACAGTTCAGAAATTAGAGCTTTTGCGACGGTGATTGAGCACCTAACAGTGCT 
Routing strand C64

BHQ1-GTACACCTTAAAGCGATTGGCACTTTAACTTTGTTAAAACTCACATTATTGAGTGTTGTTCCAGAAACC GTCTATCAGGG

Routing strand B31

AAAGAAGTTTTGCCAGCATAAATATTCATTGACTCAACATTTTCAACCAACCCTGGACACCGCATTGTACCT- FAM Routing strand C58

CTCACTGAATTGTCAAGAATCGTCCCTCGTTTTGTCGTGCACACAACATACGAGCCACGCCAGC

Routing strand C59

CAAGTTTTTTGGGGTCGAAATCGGCAAAATCCGGGAAACCTTTGCAATGGCGGTATCCTAAGGTTTCCACAC

Routing strand C52

CTTGTTGTGTTTGTTTACATGGGAAAGGTTTTCGCGCGGGCCTGTGTGAAATTGTTGGCGATTA

Routing strand C53

CTAAATCGGAACCCTAAGCAGGCGAAAATCCTTCGGCCAATTTCCACGAGCTAAATTAGACGACAGTTCGAG

Routing strand $\mathrm{C} 44$

TTAGTACATATATGATAAAACAAGATTTGTTTCCAGGGTGGCTCGAATTCGTAATCCAGTCACG

Routing strand C45

TAGAGCTTGACGGGGAGTTGCAGCAAGCGGTCATTGGGCGTTTCGTCTGTAATGTAGCCATTTCCTAAGGCA

Routing strand $\mathrm{C} 36$

BHQ1-GGCCCACGCTGATAGGTATCCTGAGCACGTTTGGCAACAGCAGTCACACGACCAGTACATTGGC

Routing strand C4

AGATTCACCTGATTGCCCTTCACCGTGAGACGTTTGCCTCGGTTTCTGTTGACTCTATTGGATC- FAM

Routing strand $\mathrm{C} 7$

ATGGAGTAGTGATGACCGATTCGTGCTTCTTTGGACATTCACCTCAAATATCAAACACAGTTGA

Routing strand C8

TTGACGAGCACGTATACTGAAATGGATTATTTAATAAAAGTTTTCGTTGTGCGAGCGAAAACACGAACTGAG

Routing strand $\mathrm{C} 15$

ACGAATCCGATTCTCCAATGTGTCCGTGTTTTTGACCTGACAAATGAAAAATCTAAAATATCTT

Routing strand $\mathrm{C} 16$

AGAATCAGAGCGGGAGATGGAAATACCTACATAACCCTTCTTTCAAGGGCGAGAATTGGAGACATATTTAAA

Routing strand $\mathrm{C} 22$

TCATCATCGATTAGATCTACTGACGTCCCTTTCAATATTTGCCTGCAACAGTGCCATAGAGCCG

Routing strand C23

TTAAAGGGATTTTAGATACCGCCAGCCATTGCGGCACAGATTTTGAGGCTGCAATTCATAGTAGCCTCCGAA

Routing strand $\mathrm{C} 27$

FAM-CATTCCTCCAAAACGCGGCAAGCCCTAGTTTTCGCGAACTAAAACAGAGGTGAGGCTTAGAAGTATT

Routing strand $\mathrm{C} 28$

GAATCCTGAGAAGTGTATCGGCCTTGCTGGTACTTTAATGTTTTTAACAACAGAGGACATCTTTTTAAAACT-BHQ1

\section{Sequence of release strands}

B58-59 CTGCAACTTGAGCAAGTATAGTGGGTGCTTATTATTATTATTATTTGGACCTCTAGTC

B52-53 GAGCTCTTCTGGTTAGAATTCGTGGTAGGTTTTTTTTTTTTTTTTGGAGCTCTTTCGA

B44-45 GGAACCGGATCTTTTGGCAATAGGGGTGAGACTGCATTAGAGTCTAGAATTGATCAGA

B36-4 CGATAAATAATTTGTCACCCTTTTCAATCTACGGATCTCATATCTGCCACAAGGAGCG

B7-8 AGTAGACGACGCACCTATGTAGGTGAGATGAGAATCCTGAATGCGATGCTACTCGTAG 
B15-16 ACGACTGCCGAAATTATGATGTCCAACACCTTTACCACGTAGTATTCAGGCGAACCCC

B22-23 GAATAATAGAAGTGTTTGGGTGCCACCTGCCCAGGAGAGCGTCCGGACACTAGAAATA

B27-28 AGCACTGTTAGGTGCTCAATCACCGTCGCTCGCCACTCTAGTCCGACCCGCTAGTCAC

B31-C64 AGGTACAATGCGGTGTCCAGGGTTGGTTGGTTAAAGTGCCAATCGCTTTAAGGTGTAC

C58-59 GTGTGGAAACCTTAGGATACCGCCATTGCCGAGGGACGATTCTTGACAATTCAGTGAG

C52-53 CTCGAACTGTCGTCTAATTTAGCTCGTGGACCTTTCCCATGTAAACAAACACAACAAG

C44-45 TGCCTTAGGAAATGGCTACATTACAGACGCAAATCTTGTTTTATCATATATGTACTAA

C36-4 GATCCAATAGAGTCAACAGAAACCGAGGCCGTGCTCAGGATACCTATCAGCGTGGGCC

C7-8 CTCAGTTCGTGTTTTCGCTCGCACAACGAGAAGCACGAATCGGTCATCACTACTCCAT

C15-16 TTTAAATATGTCTCCAATTCTCGCCCTTGACACGGACACATTGGAGAATCGGATTCGT

C22-23 TTCGGAGGCTACTATGAATTGCAGCCTCAGGGACGTCAGTAGATCTAATCGATGATGA

C27-28 AGTTTTAAAAAGATGTCCTCTGTTGTTAAACTAGGGCTTGCCGCGTTTTGGAGGAATG 


\section{Sequence of staple strands used in the assembly of the 23HB barrel DNA origami}

Staple strand 1 GAAGGCTTCAAGAACTACTAGAAACTTAATTGAGAATCGCCATAACATATATTTTGTAGATATA Staple strand 2 TTAGCGTAAAAAGCCAGCACCCTCACGAAAGACAGCATCGTCCATGTGCCGACAATCCTCATAG Staple strand 3 AATCAATACTGATAACCGAGTATGCAACAGGAAAAACGCTAACATTATAATTACAAAACCCTC Staple strand 4 GTGTAGCGCCACTACAGTCGGGAGAGAGTTGCAGCAAGCGAGTGAGGCCGCCCTAAACTGGCAA Staple strand 5 AAGTTTGAGTCATGGAACAGTTGGCACAAACATCAAGAAAAGTGAATATTGCGTATAATTTTAA Staple strand 6 CGAACGTGAATCGGAATTGGGCGCCAGGGTGGTTTTTCTTTAGTAATAATGAGGCGGAAGCCGG Staple strand 7 GTTGATATTAGGATCACCCTCAGAGCCACCACCCTCAGAGGAACAACTGGCACCATCGAGAGG Staple strand 8 TAGAAGTATGTCACACGCACTAACAACAAAATCGCGCAGAGGTTATACAATAACGTTGAGGATT Staple strand 9 ATTCAACCGATTCAAGATTCCCAATTCTGCGAACGAGTAGACATCAACAAAACGACATATGAT Staple strand 10 TGTTTAACGGCATTTTCTCCCGACACATAAAGGTGGCAACCATTTGGTAGCAATAACGATTTTT Staple strand 11 CATCGGCATCTTTTTAAGCCATTACTGAGTAACAGTGCCCGAGCCACAATGGAAAGCGCGTTTT Staple strand 12 GTTTGCCATACAATGAAAGAATTAGGCCCCCTGCCTATTTGAACCGCTATTCACAATTATTAGC Staple strand 13 GATAGCAACGGGGTGACTGTAGCGCAGTCTCTGAATTTAAGCATTCCATCAGCAGTTTTCAGG Staple strand 14 GAGCTAAACTTATAAATCTTTGACCCCTTCTGACCTGAATTAGGAGACCAGTAATCAGAGCGG Staple strand 15 ATTATAGTTTGCAAACAAAGCTGACGTTGGGAAGAAAAATCATAATGCTTTTTAGAACCTGACT Staple strand 16 AACCACCAGATATTACCCAAATATCTTTAACAATTTCATTGCTTAGACAAAATTAGAACAAAGA Staple strand 17 CCTGCAACAGTGCCAGAGTGAATAACCTTGCTTCTGTAAATCGTCAGCAAGC Staple strand 18 ACCCTCAGTATAAATAGCCCCCACAAATAAATCCTCATTCGATCTAAAGTAGCAAGAACCGCC Staple strand 19 TCTTCTGATCAGATGAATTTTTAACAATAATATCCCATCAAGCCTTAAAGGTAAATTAATTTCA Staple strand 20 CAGAGAGAATATTTAACTTCTAAGCAAAGACACCACGGAAAGGTGAATTAAGCCCCAGCCTTTA Staple strand 21 ACAGGAACGTGTTTGATTGCGCCGGGCACAGACAATATTAGGAATTGATTATTTAGATTTTAG Staple strand 22 TGTCCATCATCACCGCCTAGGGCGACATCGCCATTAAAATGAACCTGCCAGCCATAAAGAGTC Staple strand 23 CCAGAGCCAAGAATTGAGTTATCACTGAAACATGAAAGTATACTCAGAGGAGGTTGCACCGGAA Staple strand 24 CAAAGGCTTAAAAATGTAGCTCAATATATTTTCATTTGGGGGCTCATTTGATGTGCAGATCTA Staple strand 25 CCGCGCCCACTCATCATACAAATTCTTACCAGTATAAAGCCGAATTAACTGGTTTACATCATTA Staple strand 26 TTATTTATTACCGACAAAATCAAGGCAGTATGTTAGCAACAGTAGCAAAAAGTAAACAGCCATA Staple strand 27 TTACCCAAATTTCGAGGTTGTGTCGATAGCGAGAGGCTTCAGAAGCAAAAACGAACGATATTCA Staple strand 28 GCCTAATTACGACAATACTACAATAGAACTGGCATGATTAAACGTCAAGGAAACCCTTTCCAGA Staple strand 29 TCATCTTACTACGAAAAAGGAATTGCGAATAATAATTTTTAAATTGGGCCTGCGGATAAAACAC Staple strand 30 TGCTAAACAGCATTGACGAGGTTTATTTTCATGAGGAAGTCAAAGTATTAATTGTATGGGATTT Staple strand 31 AGTCAATACAAAATTCATTTTGCGTTTGCACGTAAAACAGAATAAATAAGGCTGTCCTGAGAAG Staple strand 32 CGAAAGGATTTTTGGAATCGGCCCAACAGCTGATTGCCCTCGCAAATTAACGAACCAAAGAAAG Staple strand 33 AGTTTGCCTGTTACCAGACCAATGATGGTAATAAGTTTTAAGCCCAAGTAAGCGTCCAGAATCA Staple strand 34 ACACCGGACTACCATATTTAAGACGTTTCCTTATCATTCATCCGGTAAACGCCAATAAGAATAA Staple strand 35 GATTATCAGCAAACTATAAAAATCTAATGGAAACAGTACATCCCTTATTCTGAATCATATTCCT Staple strand 36 AAAAAGATCGATAAAAAGAACCGTAACGGAACAACATTATTTTTTTGCGAAGCCTTTTTGCATC Staple strand 37 GAATAGAAAGCCGCCACAGCCCGGAAAAATACGTAATGCCTGACCCCAAAATCTCCCGGAGTGA Staple strand 38 TTTAAACATCCAATATTGAGATGGTTTAATTTCAACTTTAATAACAGTTAAGGGTGACAAATGC Staple strand 39 TAGTATCAGGATTATACGAATCCTTAAACCAAGTACCGCAATAGCAACAGTAGGGAAGCCTGTT Staple strand 40 TTAATTTTAAATCAAATTCATCAATATAATCCTGATTGTTTTATGCGTTGAGAACAGCTATTAA Staple strand 41 ATCAAGAGTATAGTTGCTACTTAGCCGTTTACCAGACGATAAGAGGAAAGATTCATTGACCTTC Staple strand 42 TGGAGCAAGCGGGAGGATGGCTTAGCATCAATTCTACTAATTTGTTAAACTTCGCTGAGAGTC 
Staple strand 43 AGGGAGGGATTCATATGAACACCCTGAACAAAGTCAGAGGGCAGAACCGCTAGCGGGGCCGATTG Staple strand 44 ATCGTAAACCAAAAATAATTGCTCCCAATAAATCATACAGGATAAGCAAACTGTTGAACGGTA Staple strand 45 AGAGGTCAACAGGTAGAAGCCCGACCCTGTAATACTTTTACAAGAGAATTAACATCTTTTGATA Staple strand 46 ACCCTCACGGAACCATCAAAATAGGCAGGTCAGACGATTTAAATGAATAAAGACTGTACCGCC Staple strand 47 ATTCCATATCATTGTGAAACGAGATAATGTGTAGGTAAAGTTCTAGCACCATTAGGGAAGTTTC Staple strand 48 CGCCACCCTTAATTGAGCTATTGACTCCTCAAGAGAAGGATAAGTATCAGAACCACCTCAGAGC Staple strand 49 TCTGAGAAAAGAAGAGAACGTTATGATTTTCAGGTTTAACGCCTAAATTACGAGCAATCATAGG Staple strand 50 GATTAGAGACCACATTCATTCGAGGCTAAATCGGTTGTAACTAGCATGAATTAGCACAGGTCAG Staple strand 51 TTCTTTGATTTCACCAGGGAAGGGCCAGCAGAAGATAAAGCAAATGCGGCCTTGCAGCAATAC Staple strand 52 AATATCTAGCGTAAATTAAAGGCATTGGCAGATTCACCATAGACTTTAATTCATTTTATCTAA Staple strand 53

AAGTGCCGACCTAAAACGAAAGAGGCAAAAGAATACACATCGTCATAAATATTCATTGAATCCCCCTGAAAGGCC Staple strand 54 CGGAGAGGAATGCAAACGGTGTCTATACATTTCGCAAATGGATAATTCGCGCCAGGTTAATGC Staple strand 55 GCGCGTACCTATTAATAAAGCCTGAAATCGGCAAAATCCCAGGAGGCCGGAATACGTCTACAGG Staple strand 56 GGCGTTTTAATAATCGGCGTTAAACATGTAATTTAGGCAGAGTCAAAAAAAGAAACGAACGCGA Staple strand 57 ACCTTGCATACCGAACCGTTGTTGGTAATATCCAGAACAAAGGAGCGGCTTTTTTAAAGCATC Staple strand 58 TGCTGAATTACGTTAATAAGCGGAATTTCAACGCAAGGAATCAGGTCAAAAGGTGGAGCTTAAT Staple strand 59 AGGTTTTGCTAATTTTAATGGTTTAATAAGAGAATATAAAGCCCAATCCAAATACATTTGCGGG Staple strand 60 GAAAACTTCGGGAGAAATAACTATACAACAATAGATAAGGCACCCAGAACAACATAGAACGCGA Staple strand 61 TGTACAGACACGCATAAATCATAAGATAGTAAGAGCAACGCGTTTTAAACTAATGCATGAACGG Staple strand 62 AGACGTTAGGGCCTTGACACCCTCACGGCTACAGAGGCTTTGATAAATGAATTTCTGTCTTTCC Staple strand 63 GCCAGCAACAGAGGCATCACTTGCCTGAGTAGAAGAACTATGATGGCATATATGTCGCTGAGA Staple strand 64 CGCGAAATTCCATTAAGTTTCAGAAAAAAAAGGCTCCAAAGCTTGCCCTAAATGTTATACCAAG Staple strand 65 GGCGCAGCGTCACCCCAGACAGCGACAACAACCATCGCCCCAGGCGCATTAACCCTCGGAACGA Staple strand 66 GTATTAAATCTGAAATGGAGGAAGGTCAATTACCTGAGCAGACTACCTATACAGTCGACAACTC Staple strand 67 GCTATTTTTCCTGAAATATTTTAGGTAATTCTGTCCAGACGTGCCAGTTCTTATTACATTAGTT Staple strand 68 TAAATCAAAATAGTAGACGAGAATGCGATTTTAAGAACTGGACTAAAGTTGCCTGAGATGACCA Staple strand 69 ATCACCGTTAAGAGCCGCCTCCCACCAGAGCCGCCGCCAACTTTCAACAACGGGTATAGGTGT Staple strand 70 ACCGTGTGAATAAAGAATTATCAAATGTAGAAACCAATCAGCGAACCGAGCCAGTGAAATACCG Staple strand 71 AACGCCTGTCCGTTCCATAGGAACCCCGCTTTTGCGGGATACGGTCACCGATATATCAAACTAC Staple strand 72 GTGAATAAGAGGAGCCTCAACGGAGTTGCCAGAGGGGGTAAATCAGGCCAGTCAGGCTCATTCA Staple strand 73 AGACGGGAAACGCTCAAGCAAATCCACAATCAATAGAAAAAGGTAAAGCTAATATAAGCGCATT Staple strand 74 CATCGCCTGAGGACTTTTCTGTATCGGTTTATCAGCTTGCTCAACGTAAAGAAGTTATTTGTAT Staple strand 75 TAGGTTGGGCGAATTCAAACAATTAACAGTACCTTTTACATTTTCAAATCAAGAAACTCCGGCT Staple strand 76 AGCGATATGAATTACAATTATCATAATGGAAGGGTTAGAACATCATAATGGGTATTGAAAACAT Staple strand 77 AGTTGAATTTGAATAATCCTGATTTTGACGCTCAATCGTCCTTTGCCCTGATGAAAAATCAAC Staple strand 78 TTTATAATCGTCCACGCGCGCGTAGTCTTTAATGCGCGAATCTGGTATACCTACAGAAGTGTT Staple strand 79 CAAATATCACTATCAAGGCTGGCCAGTTGAGATTTAGGAATAGTACCTTCATTATGAAAGACTT Staple strand 80 CACCCGCCGGGCGAAACATTAATCCAGCAGGCGAAAATCCGTACGCCAGGGCTATTAACCACCA Staple strand 81 AATATGCACTCATTATATCTTTACCCCTCATATATTTTAGTAGCTATTGTTTAGCCATGTTTTA Staple strand 82 ACGAGTAGTTCACGTTGCAGCGATTTAGACTGGATAGCGGTTCAGAAATTACCTTAACACCAGA Staple strand 83 GACCTGCGAACGAGGGTTTTGTCTAAACAGCTTGATACCGAATCTTGACAACCAAAAAATCCGC Staple strand 84 CATCAAAATCAATAACCTTTTGAGTGCAAGGCGATTAAGGCCATGTTAGGTCACTAGGAACGC Staple strand 85 GTCCGTGAGATGCCGGCAAACGCGCGGTGGTGCCATCCCACCCGCTTTCCGTGAACCATCTTCGC 
Staple strand 86 GTACAGCTTGGGTAACGTCTGGCCCAAACGGCGGATTGACCGTCGCTGGCCTGCATGGCGAAAC Staple strand 87 AAGATTGTCAAGGCAAAGTCAATCCCATTCGCCATTCAGGGATCAATCCGGCACAAAAACAGG Staple strand 88 GCTGATTGCATCGTAACCTTTCTCCATCGTTAACGGCATCAGCCTCCCTTACGGCTTTAAACGAT Staple strand 89 GGGAGAGGCTGGTGCTGTCATACCTGCCGTAAAGCACTAGCGAGAAATGAGACGGGAACGCGCG Staple strand 90 TTGTGAGGAAAGGGGTTTAACCAAGTTGGTGTAGATGGGCGCCGTTCCGGGTTACCCCCGGAAT Staple strand 91 TCTGCCACGGTGCCCCAGCCTCCGGCAGCAACCGCAAGAATGCATTAATGGGTCGAGGGGGGGTT Staple strand 92 CATGGTCTTCTTTGCTAAAAAAAGGCTTTCGCACTCAATCCCATAAAGTGAGAACGTGTCGTAAT Staple strand 93 GTGCCAGCTGCCAACGGTGCCTGTTCACCCAAATCAAGTGCGGGCGCTGGCCCTGAAACCTGTC Staple strand 94 TTAATATTAGTAGTAGCATCGATGGGAAGGGCGATCGGTCTCTCACATCGGCCTTTGTAAACG Staple strand 95 GGCCGTTAGTGTCACCGTGCCGGACTTGTAGAACGTCAGCGGGTTTGCGTACCCTAAATGCGGCG Staple strand 96 CGGCCTTTGACGACAGTGGAAAAAGAAACATCCCTTACACTACCGAGATTGCAGGCCCGCACAGG Staple strand 97 AAACAGCGCTGCGCAATATTTAAACAGGAAGATCGCACTCCAAATCCCGTCGTCATAGACGCAG Staple strand 98 CCAGCTTTTTTAGTTTGTGATAAAGTTTTCCCAGTCACGTCACCGGCGTGGGAATTCCTGTAG Staple strand 99 TTAAATCACGCGAGCTGATTGCCTATTACGCCAGCTGGCAGATAGACGTGCATCAAATTTTTG Staple strand 100 GGGATAGGCGGGCCTATTCGCATTTGCCAGTTTGAGGGGACAGTGATGATCAGCAAGTGGTGAA Staple strand 101 CATCCTCACGGATTCTCAAACAATCCAGACGATCCAGCGCTTCACGGGTCTGGTCAGCCAGAGCA Staple strand 102 GCTCACTGCGCAACCAGTCACAGTTATCAGGGCGATGGCGTCACGCTTGGTTTGCCTGCGTTGC Staple strand 103 TGCTGCGGCCAGAAGGGAGCCCCCGATTTAGAGCTTGACGGGGATCAGTATTAACACCG Staple strand 104 AGCCGGAAGGCCGGGCGTTTCCTGTGGAACAAGAGTCCATATGGTTGCAAAAGAATAACATACG Staple strand 105 CCCCGGGTGGTGTGTAGGGTAAAGGGAGGTGTCCAGCATCAAGCTAACTCAAACCGTCTGAGGAT Staple strand 106 TAATGAGTGGCGGGGTCCTCGAATGACTCCAACGTCAAAGCGCTTAAGGTGGTTCCGGGGTGCC Staple strand 107 ACATAAAAAGCCAGCTTACTTAAATGTAATGGGTAAAGGTATAGCTGCGGTTGCGGTGTACATCG Staple strand 108 GGAGACAGTCAAATCACCATCAGGCCAGTGCCAAGCTGAGGTGGAGCCGCCACTGTGCACTCTGTGG Staple strand 109 TTCGTCTCGTAATGGGATTACCAGTTGCAGCCAGCGGTGCGCACGCGCAGCACCGTGTCCGTTTT Staple strand 110 GATAACCACGTTGTATTAAATGTGAGCGAGTAACAACCCGTTAACGGAATGCGCGCCGGGAACG Staple strand 111 CCAACGCTTACCCAAATTTATCCAATGCAGAACGCGCCGCAAGACAAGTTCAGCTTGAATCTTA Staple strand 112 CGCTCACAATTCCAGTTTGTGAAAGGTCACTGTTGCCCTGGGCGGTTGTATGAGCCGTTGTTATC Staple strand 113 GCTTTCCTCAGATAGAAGAGCACGTAGGGTTGAGTGTTGTTCCACACAGCCCGAGATATAACGT Staple strand 114 ACTTTGAAAACGAGGCGGAACCGAGAGGCTTGCAGGGAGCACCAGTATCGGTCGCTACTGACCA Staple strand 115 ACCGGAAGAGCATAAACTTCAAAACGCCAAAAGGAATTGAGGACAGAGATACATAGCGAACCAG Staple strand 116CGTCAATAGCCAAGTTACTAATAGATTCTGGCCAACAGGTTAGAATAAAAGGGACATTAGAGC Staple strand 117 GAGTTTCGTTTAAAGGCATGTACCTTTTGATGATACAGAGTAGCGAATACATGGCGTAACACT Staple strand 118 ATCCAATCTGTTTATTGTAAATGTGATTGCTTTGAATAATAATACATGATTCGCCCTGATGCAA Staple strand 119ATAATCAGCAAAGCGATATGTAGCAATAAAGCCTCAGCAAACTCCAAAAATTAACCCCGGTTG Staple strand 120CAGCAGTTGCGGCTGTTCTGCTCGTGCCGGAAACCAGGAAAAGCCCCCGCTTCTGATTTGCCGC Handle strand 1 GGGCGACATTCAATTTTGCTCAGTACCAGGCGGAT Handle strand 2 CGTTTTTATTTTCATCGTAGGACAGCGCCAAAGACAAAA Handle strand 3 ATTCATTAATAAGTTAAAACAGGGCAGAGAGA Handle strand 4 TAACCCACACCACCGGAAGCTGAGACGGAAATT Handle strand 5 GACTTGAGCATATAATGAAAATAGAATAATAA Handle strand 6 GAGCAAGAACTTTTCATATATTATTCCGTCACC Handle strand 7 AAAATCACACGTAGAAAATAAGAAGCTATCTT Handle strand 8 ACCGAAGCCTTTCGGTCACAGTTAATAGCCAGC Handle strand 9 AAGGCCGGAAGACTCACAAAATAAGCAGATAG 
Handle strand 10 ATAATAACGGAAAACGAGCGTGAGGAAACGATA

Handle strand 11 GCAGCACCGTAATCGAGTGTACAACCATCGCA

Handle strand 12 CCGAACAAATTAGCGTCACAGTGCCTCATTAGC

Sequence of capturing strands and routing strands used to replace corresponding handle staple strands in the assembly of the $23 \mathrm{HB}$ barrel DNA origami (red: $40 \mathrm{nt}$ or 20 nt long extensions of strands for binding RNA; blue: 15 nt long extensions of strands for toehold exchange reaction to release RNA)

Handle strand 1

TATGGTCGACCTGCAGGCGGCCGCACTAGTGATTGGGAGAGGGCGACATTCAATTTTGCTCAGTACCAGGCGGAT Handle strand 2

CGTTTTTATTTTCATCGTAGGACAGCGCCAAAGACAAAAGACGAATTCGAGCGCACCACGTGTGATTACGGACACAATC Handle strand 3

AGTTGCAGGACTAGAGGTCCACGCAATAGACGGACATTCATTAATAAGTTAAAACAGGGCAGAGAGA Handle strand 4

TAACCCACACCACCGGAAGCTGAGACGGAAATTAACGGTAAGAGGCTCCCAAACCAGAAGAGCTCTCG

Handle strand 5

TCCGATTGTAAGATCCGGTTTTCCACGTAGGACGGGACTTGAGCATATAATGAAAATAGAATAATAA Handle strand 6

GAGCAAGAACTTTTCATATATTATTCCGTCACCACTCTCTCCATAAGGCTGATCAATTCTACTATTAA

Handle strand 7

TAACGTTTCGGCAGTCGTGGACAACACCTGACCTAAAAATCACACGTAGAAAATAAGAAGCTATCTT

Handle strand 8

ACCGAAGCCTTTCGGTCACAGTTAATAGCCAGCCGGATGGCGTTACAATCGCCTGATTTTCAACTTCT

Handle strand 9

ACCGCATTGTACCTGTACACTGGACTAGCCAGGAGAAGGCCGGAAGACTCACAAAATAAGCAGATAG Handle strand 10

TTCGAGCTTGTTGTGTTTGAAGTAGTAGTAGTAGTATAATAACGGAAAACGAGCGTGAGGAAACGATA Handle strand 11

AGATGGAGTAGTGATACTATAAACGATGCTGAAGGGCAGCACCGTAATCGAGTGTACAACCATCGCA

Handle strand 12

TTCCTCCAAAATCCTTAACACTCAGGTTTCACAGGCCGAACAAATTAGCGTCACAGTGCCTCATTAGC

\section{Sequence of release strands}

Release-handle strand-3 GTCCGTCTATTGCGTGGACCTCTAGTCCTGCAACT

Release-handle strand-4 CGAGAGCTCTTCTGGTTTGGGAGCCTCTTACCGTT

Release-handle strand-5 CCGTCCTACGTGGAAAACCGGATCTTACAATCGGA

Release-handle strand-6 TTAATAGTAGAATTGATCAGCCTTATGGAGAGAGT

Release-handle strand-7 TAGGTCAGGTGTTGTCCACGACTGCCGAAACGTTA

Release-handle strand-8 AGAAGTTGAAAATCAGGCGATTGTAACGCCATCCG

Release-handle strand-9 CTCCTGGCTAGTCCAGTGTACAGGTACAATGCGGT

Release-handle strand-10 ACTACTACTACTACTTCAAACACAACAAGCTCGAA 
Release-handle strand-11 CCTTCAGCATCGTTTATAGTATCACTACTCCATCT

Release-handle strand-12 CCTGTGAAACCTGAGTGTTAAGGATTTTGGAGGAA 


\section{References}

[1] Rothemund, P. W. K. Folding DNA to create nanoscale shapes and patterns. Nature 2006, 440, 297-302.

[2] Zhou, K.; Ke, Y.; Wang, Q. Selective in situ assembly of viral protein onto DNA origami. J. Am. Chem. Soc. 2018, 140, 8074-8077.

[3] Fraenkelconrat, H. Degradation of tobacco mosaic virus with acetic acid. Virology 1957, $4,1-4$.

[4] Butler, P. J. G. The current picture of the structure and assembly of tobacco mosaic virus. J. Gen. Virol. 1984, 65, 253-279.

[5] Ke, Y. G.; Lindsay, S.; Chang, Y.; Liu, Y.; Yan, H. Self-assembled water-soluble nucleic acid probe tiles for label-free RNA hybridization assays. Science 2008, 319, 180-183. 